\title{
Robust Design of Transonic Natural Laminar Flow Wings under Environmental and Operational Uncertainties
}

\author{
Christian Sabater* Philipp Bekemeyer ${ }^{\dagger}$ and Stefan Görtz \\ German Aerospace Center (DLR), Institute of Aerodynamics and Flow Technology, 38108 Braunschweig, Germany
}

The introduction of laminar flow configurations is envisioned to provide new opportunities to further reduce aircraft fuel consumption. The robustness of laminar wings is critical, both against instabilities that can unexpectedly trigger transition and against off-design conditions outside the cruise point. However, current inverse design methodologies not only provide suboptimal configurations, but are unable to come up with robust configurations. The objective of this paper is the development and demonstration of a framework for the robust direct design of transonic natural laminar flow wings using state-of-the-art industrial tools such as computational fluid dynamics, linear stability theory and surrogate models. The deterministic optimization problem, which serves as a baseline, searches for the optimum shape that minimizes drag applying a surrogate based optimization strategy. In that case Cross-Flow and Tollmien-Schlichting critical N-Factors are fixed according to calibration data. For the robust approach, uncertainties in these critical $\mathrm{N}$-Factors as well as operational conditions such as Mach number are considered to account for situations that could prematurely trigger transition and thus significantly decrease performance. The surrogate based optimizer is therefore coupled with a surrogate based uncertainty quantification methodology, following a bi-level approach. The objective function shifts towards the expectation of the drag to minimize average fuel consumption, or the $\mathbf{9 5 \%}$ quantile to account for extreme events. The framework is able to come up with state-of-the-art natural laminar configurations for a short-haul civil aircraft configuration. The deterministic optimum is able to delay transition till $60 \%$ of the wing upper surface where the shock is present but is highly sensitive to small changes in the predefined critical $\mathrm{N}$-Factors, as minor deviations will lead to fully turbulent configuration and hence an increase in drag. The robust configurations are more balanced, as the transition location smoothly moves upstream as the critical N-Factors are reduced. As a direct consequence, obtained pressure profiles are more resistant against instabilities, extending the current design envelope of natural laminar flow wings.

\section{Nomenclature}

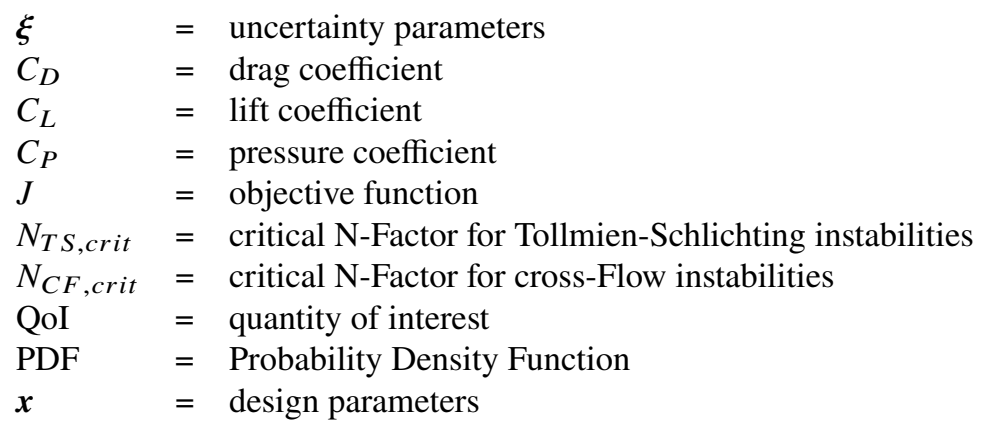

\footnotetext{
${ }^{*} \mathrm{PhD}$ student, $\mathrm{C}^{2} \mathrm{~A}^{2} \mathrm{~S}^{2} \mathrm{E}$, Lilienthalplatz 7, AIAA Member

${ }^{\dagger}$ Team Leader, $\mathrm{C}^{2} \mathrm{~A}^{2} \mathrm{~S}^{2} \mathrm{E}$, Lilienthalplatz 7, AIAA Member

${ }^{\ddagger}$ Department Head, $\mathrm{C}^{2} \mathrm{~A}^{2} \mathrm{~S}^{2} \mathrm{E}$, Lilienthalplatz 7, AIAA Member
} 


\section{Introduction}

With the continuous growth of commercial aviation, environmental and economic concerns are key drivers towards the reduction of operational cost and emission of greenhouse gasses [1]. Although the aerodynamic design of transonic wings is already a mature field, the introduction of natural laminar flow (NLF) configurations can lead to new opportunities to further reduce fuel consumption [2, 3]. Laminar boundary layers present lower shear stress at the wing surface, reducing viscous drag and improving aerodynamic efficiency.

Historically, only low sweep wings flying at reduced Reynolds number have benefited from natural laminar flow [4]. At higher Reynolds numbers and sweep angles, crossflow (CF) instabilities are triggered that lead to boundary layer transition near the leading edge [5] causing a fully turbulent flow over the wing. The application to larger Reynolds/sweeps usually requires the combination of NLF with laminar flow control such as suction [6] of the boundary layer to dampen instabilities. However, it is possible to obtain pressure profiles that mitigate $\mathrm{CF}$ instabilities through shape design without the need for active flow-control devices. An example for the redesign of the NASA Common Research Model can be found in [7, 8] applying inverse design techniques.

Currently, inverse design methods based on linear stability theory are used to design laminar wings in research environments [7, 9]. Geometries are found following prescribed target pressure distributions that are expected to maintain laminar flow. This highly relies on the aerodynamic expertise of the designer. Figure 1 represents the typical inverse design process. The pressure distribution should have an initial acceleration at the leading edge to mitigate $\mathrm{CF}$ instabilities, followed by a favourable pressure gradient that dampens Tollmien-Schlichting (TS) instabilities. Transition should occur as downstream as possible on the upper surface to maximize the extent of laminar flow and consequently minimize viscous drag. Then, a shock wave is required to decelerate the flow. The end of the wing is shaped in order to provide rear loading allowing to meet lift requirements. In practical applications, laminar flow can only be maintained over the upper surface of the wing and early transition occurs on the lower surface of the wing due to the integration of leading edge Krueger slats. After the desired pressure distribution for a given flight condition is defined by an expert, inverse design methods are used to find a shape that matches the target pressure as close as possible. Finally some iterations by hand take place aiming for a more detailed design in the leading and trailing edge regions, as well as to account for any constraint in the shapes. However, this process most likely leads to suboptimal configurations. As an alternative, direct optimization approaches are able to better explore the design space. A well-posed direct optimization problem should be able to come up with at least similar pressure distributions, and more often than not, outperform inverse design methodologies.
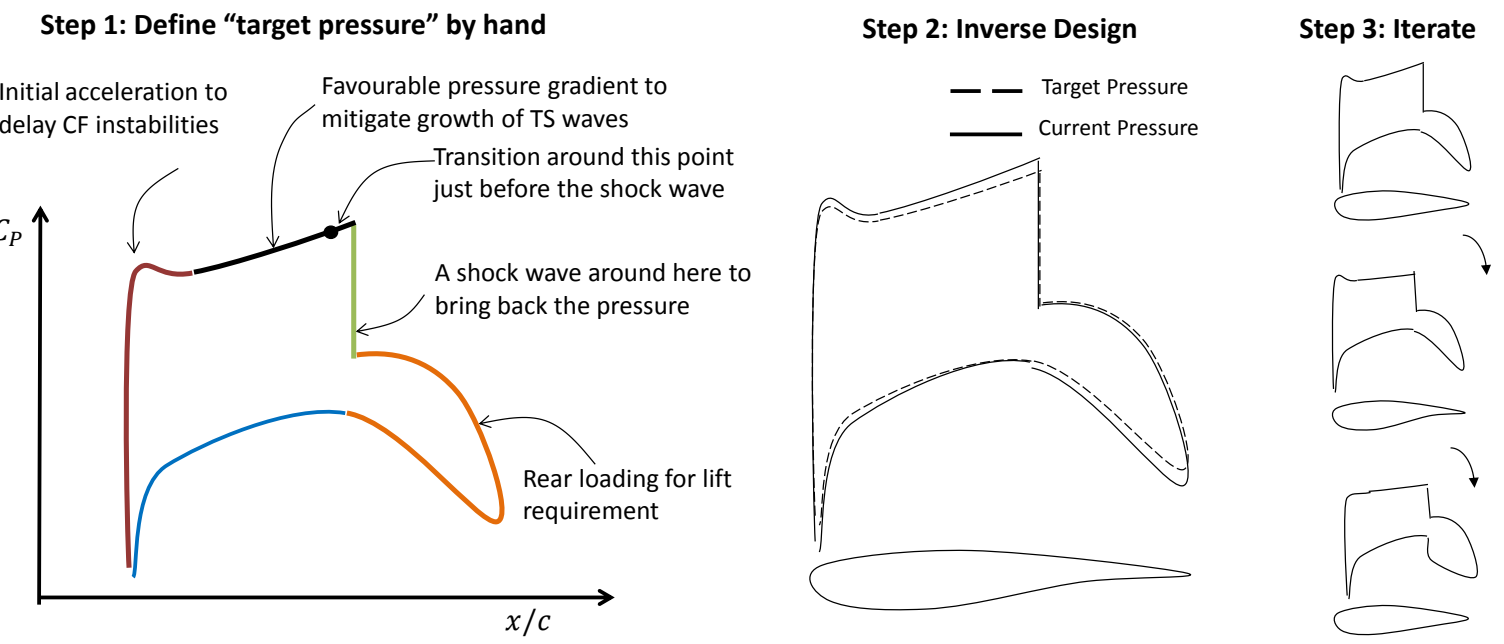

Fig. 1 Traditional inverse design process: Definition of target pressures following physical insight, inverse design to obtain the required pressure distribution and iteration to obtain the desired behaviour

The extent of the laminar flow regions strongly depends on the environment [8]. Hence, the robustness of laminar wings against environmental changes, causing instabilities that can unexpectedly trigger transition, and off-design conditions is crucial. Designing robust laminar configurations with an inverse method is simply not possible. Not only because targeting a pressure distribution can initially lead to suboptimal configurations but also it will not necessarily lead to a good design in case of uncertainties in freestream turbulence intensity, manufacturing tolerances, wear and tear, 
acoustic waves or presence of clouds. These uncertainties will effectively reduce the critical N-Factor used to predict the transition location and transition will be shifted upstream. This problem is present not only in free flight but can also be observed during wind tunnel testing [8]. For example, wind tunnel tests performed at the National Transonic Facility in NASA Langley estimated a critical N-Factor between 4 to 8 for transition caused by TS instabilities [10].

Aerodynamic shape optimization is a well established discipline even in industrial environments [11]. Lately, direct shape optimization has been used for the design of natural laminar flow airfoils and wings [12]. NLF airfoils using the $R e-\gamma$ turbulence model have been robustly designed in [13] for a range of Mach number and lift coefficients. In that case, a linear combination of mean and standard deviation of the drag coefficient was minimized. In [14], a supersonic wing-body laminar configuration was robustly optimized in Euler flow in combination with a boundary layer solver. The value at risk (quantile) and conditional value at risk were obtained under uncertainties in the geometry and one critical $\mathrm{N}$-Factor. More recently [15], robust design of laminar airfoils under uncertain critical N-Factor was performed using the low-fidelity tool XFOIL, an integrated boundary layer solver in combination with an $\exp N$ transition model. In that case, changes in the critical N-Factor were assumed to directly change the transition location according to the envelope of the transition waves without the need of recomputing the pressure profiles.

There is a lack of realistic applications of robust design methodologies applied to expensive, relevant NLF problems including uncertainties in both operational conditions and critical N-Factors for swept-wing flows. Previous investigations either relied on low-fidelity solvers to speed-up convergence or only accounted for 2D instabilities, limiting its applicability for swept wings. The first objective of this paper is the development of a probabilistic framework for the robust design of transonic natural laminar flow infinite swept wings using industrial CFD frameworks in order to come up with shapes with practical relevance. Realistic configurations must be robust under random operational and environmental uncertainties that could otherwise trigger transition more upstream than expected. The second objective is to shift the design envelope of NLF configurations by designing robust laminar wings for a short-haul civil aircraft with high sweep and Reynolds number.

Section III introduces the optimization under uncertainty framework that utilises surrogate models to obtain the final solution at a reduced CPU time. Section IV presents the problem formulation for NLF robust design, the applied numerical model, the transition prediction methodology and the treatment of uncertainties. Then, the optimization results are introduced in Section $\mathrm{V}$. The classical deterministic optimum serves as a reference configuration and will later on be compared to robust solutions under different input distributions. Finally, conclusions are presented in Section VI.

\section{Optimization Under Uncertainty Framework}

Global optimization under uncertainty problems require a large number of function evaluations to find the global robust minimum [16]. Next, we briefly re-introduce a framework which was previously successfully applied for aerodynamic robust design [17]. Computational efficiency is achieved by relying on surrogate models.

\section{A. Problem Definition}

Let $Y \in \mathbb{R}$ be the Quantity of Interest, QoI (performance measure to optimize), depending on design variables $\boldsymbol{x} \in \mathbb{R}^{d}$ at given operating conditions $\boldsymbol{A}$. The deterministic optimization problem aims to find the optimum set of design variables $\boldsymbol{x}^{*}$ at constant operating conditions while meeting $k$ constraints.

$$
\begin{aligned}
\boldsymbol{x}^{*} & =\underset{\boldsymbol{x}}{\arg \min }\left\{Y\left(\boldsymbol{x}, \boldsymbol{A}_{\mathbf{0}}\right)\right\} \\
\text { s.t. } & = \begin{cases}g_{i}(\boldsymbol{x}) \leq 0, & i=1,2, \ldots k \\
x_{L}^{j} \leq x^{j} \leq x_{U}^{j}, & j=1,2, \ldots d\end{cases}
\end{aligned}
$$

The robust optimization involves the presence of uncertainties $\xi \in \mathbb{R}^{m}$ either in operational or environmental conditions. As a result, the QoI becomes a random variable and the problem is shifted from the optimization of the QoI towards the optimization of an statistic of the QoI. On the one hand we consider the minimization of the mean value of the QoI, $\mu_{Y}$, as a natural choice from a cost-effective point of view. 


$$
\begin{aligned}
\boldsymbol{x}^{*} & =\underset{\boldsymbol{x}}{\arg \min }\left\{\mu_{Y}(\boldsymbol{x}, \boldsymbol{\xi})\right\} \\
\text { s.t. } & = \begin{cases}g_{i}(\boldsymbol{x}) \leq 0, & i=1,2, \ldots k \\
x_{L}^{j} \leq x^{j} \leq x_{U}^{j}, & j=1,2, \ldots d\end{cases}
\end{aligned}
$$

On the other hand we aim for the minimization of the 95\% quantile of the QoI to minimize extreme events, instead of a more conservative min-max optimization. The optimization of a given quantile is a flexible approach as it provides optimum performance with controlled probability.

$$
\begin{aligned}
\boldsymbol{x}^{*} & =\underset{\boldsymbol{x}}{\arg \min }\left\{q_{95 \%, Y}(\boldsymbol{x}, \boldsymbol{\xi})\right\} \\
\text { s.t. } & = \begin{cases}g_{i}(\boldsymbol{x}) \leq 0, & i=1,2, \ldots k \\
x_{L}^{j} \leq x^{j} \leq x_{U}^{j}, & j=1,2, \ldots d\end{cases}
\end{aligned}
$$

\section{B. Bi-Level Surrogate-Based for Optimization under with Uncertainty}

The bi-level surrogate model has been previously applied to the robust design of shock control bumps [17], but is a general-purpose framework for handling expensive black-box problems that feature a moderate number of dimensions and uncertainties. It combines a surrogate-based optimization framework as outer layer, with a surrogate-based uncertainty quantification (UQ) framework in the inner layer, as shown in Figure 2 . The outer level (red surface) handles the optimization which depends only on the design variables $\boldsymbol{x}$ and has the statistic of the QoI as output (the mean or the quantile). The inner level (blue surfaces) is responsible for the uncertainty quantification process of the QoI at a given design point $x_{i}$ under uncertainties $\xi$. In aerodynamic shape optimization, the QoI is usually the drag coefficient but might also be maximum lift coefficient during high-lift investigations.

This gradient-free framework is especially useful in cases where the adjoint solution is not readily available to provide the gradients, as it is typically the case for CFD simulations involving transition prediction with linear stability theory. By further refining the region where the robust solution is located, the efficiency of the framework compared with other optimization approaches is improved. Another advantage is the use of a parallel approach for the evaluation of the design of experiment (DoE) stage in the UQ level. As the initial samples are defined beforehand, all of them can be computed at the same time. In addition, if the black-box solver provides an in-build parallelization, an additional level of parallelization is possible. The two stages of the bi-level surrogate approach are briefly explained next.

\section{Surrogate-Based Optimization}

The outer layer of the bi-level approach is the Surrogate-Based Optimization (also known as Efficient Global Optimization or Bayesian Optimization). The main objective is to make the optimization more efficient by reducing the number of function evaluations required from the expensive black-box problem [18]. A surrogate model that is cheaply evaluated is built following the parametrization and the QoI. The Surrogate-Modelling for Aero-Data Toolbox (SMARTy) developed by DLR is used for the initial DoE sampling and for the creation of the Gaussian Process Regression (GPR) surrogate [19]. After an initial DoE in the design space using Sobol sequences [20], a Gaussian Process model is constructed that covers the design space. Then, two active infill criteria, namely Expected Improvement and Trust Region approach [18] are used to find the optimum.

\section{Surrogate-Based Uncertainty Quantification}

For the determination of both statistical moments and quantiles of the QoI, the classical stochastic approach of Monte Carlo sampling is too expensive [21]. The QoI is instead approximated at each iteration of the optimization with a surrogate model that is cheaper to evaluate using Monte Carlo [22]. An initial DoE is constructed in the stochastic space and afterwards an infill strategy is used to increase the accuracy of the surrogate. The infill criteria depends on the statistic to obtain. 


\section{SURROGATE BASED OPTIMIZATION}

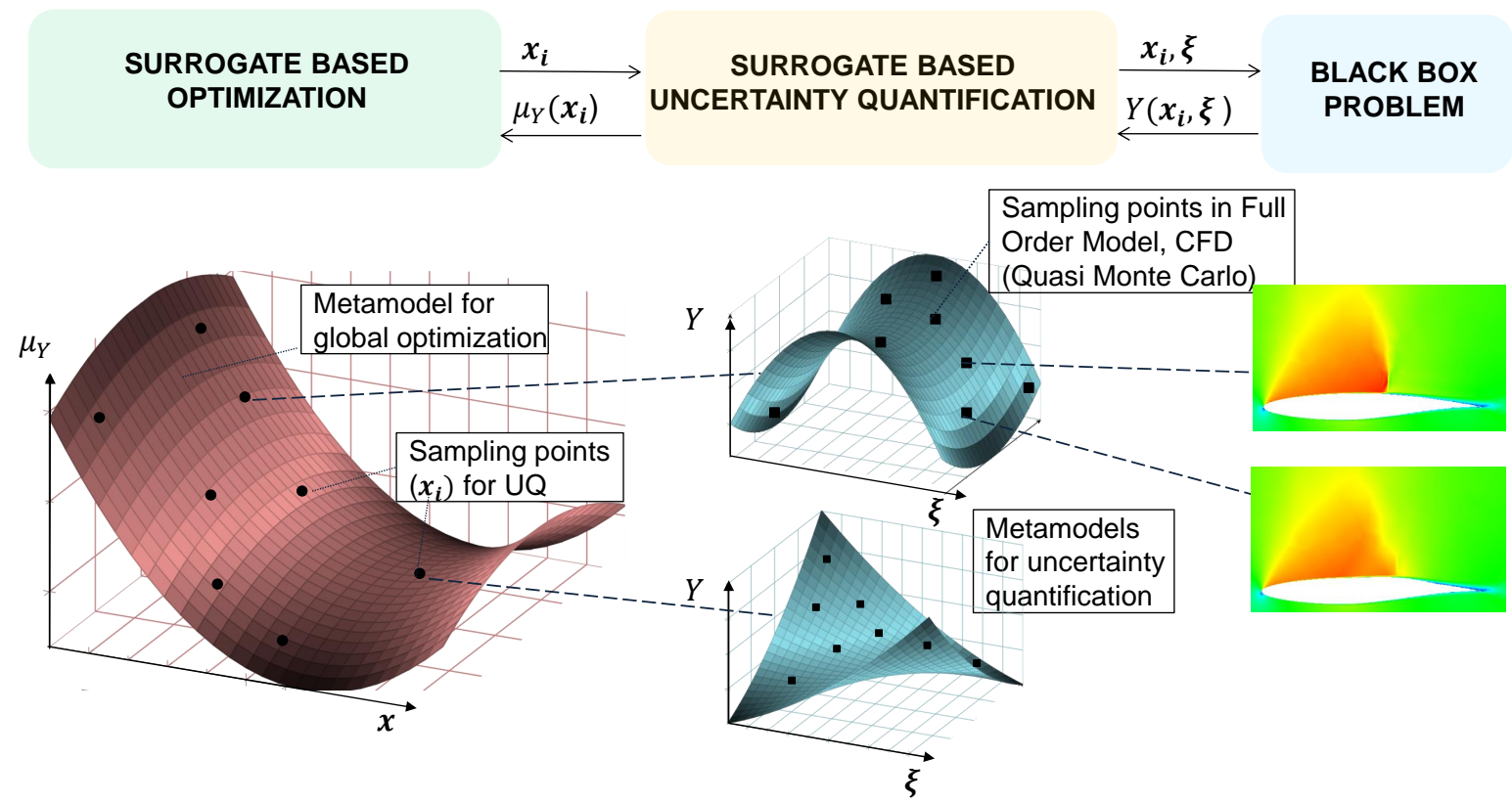

Fig. 2 Bi-level approach for uncertainty quantification [17]; Surrogate of statistics (left); Surrogate of random variable (middle); Full order model evaluation (right)

\section{Active Infill for the Mean}

If the mean value is of primary interest, a high global accuracy of the surrogate is required. To increase the surrogate accuracy with respect to the mean value, after the DoE stage, an active infill criteria that ensures an even sampling in the stochastic space [23] is used. Therefore, the prediction mean square error available in GPR at any given point in the stochastic space, $\hat{s}(\boldsymbol{\xi})$ is used. New samples are added in the location $\boldsymbol{\xi}^{*}$ where the product of the probability distribution function of the input parameters, $\mathrm{PDF}_{\xi}$ times the error estimate is maximized. The acquisition function consists on:

$$
\boldsymbol{\xi}^{*}=\underset{\xi}{\arg \min }\left\{-\mathrm{PDF}_{\xi}(\boldsymbol{\xi}) \hat{s}(\boldsymbol{\xi})\right\}
$$

The optimum location is found in the surrogate through Differential Evolution. Equation (4) balances two terms: the first one favors locations with a high PDF of the input parameters, that is sampled very often in the surrogate. The second one favors locations where the surrogate accuracy could be further improved.

\section{Active Infill for the $95 \%$ Quantile}

When looking for a high quantile such as the $95 \%$, the active infill criterion follows the U-function [24] and equivalent probability of misclassification $P_{M}$ [25]. At any given point, $\xi$, where the GPR mean value prediction is lower than the current quantile using the surrogate, $\hat{y}(\xi)<\hat{q}_{\tau}$, it is possible to obtain the probability of exceeding this quantile value, $P_{M}=P\left(\hat{y}(\xi)>\hat{q}_{\tau}\right)$. The opposite, the probability of overestimating the quantile given that the surrogate mean value is higher than the quantile, can also be obtained. The probability of misclassification is maximum when the so called $\mathrm{U}$ function is minimum. Additional samples should be added when:

$$
\boldsymbol{\xi}^{*}=\underset{\xi}{\arg \min }\{U(\boldsymbol{\xi})\}=\underset{\xi}{\arg \min }\left\{\frac{\left|\hat{q}_{\tau}-\hat{y}(\boldsymbol{\xi})\right|}{\hat{s}(\boldsymbol{\xi})}\right\}
$$

The infill criterion balances exploitation with exploration by sampling points closer to the calculated quantile or with a high error [24]. 


\section{Robust Design of NLF Wings - Problem Definition}

This section introduces the formulation of the deterministic and robust NLF wing optimization, the numerical model and the CFD process chain including transition prediction and the parametrization of the design parameters and uncertainties. The classical deterministic problem setup serves as reference solution and will be compared with the robust solution.

\section{A. Shifting the Current Design Envelope: Flight Conditions for Optimization under Uncertainty}

Both the deterministic and the robust formulations aim to design a NLF wing for a short-haul civil aircraft configuration with respective design conditions: Mach number 0.78, lift coefficient 0.7, sweep angle of 27 degrees and Reynolds number of 23 million. Following Figure 3 a practical limit for NLF configurations was established more than fifteen years ago with the Fokker 100 and ATTAS flight tests [26, 27]. Higher sweep angles and Reynolds numbers lead to premature transition due to attachment line and crossflow instabilities. The former is critical near the root of the wing due to the increased Reynolds number and contamination from the fuselage, and can be avoided with anti-contamination devices such as the "Gaster bump" [5]. Crossflow instabilities can be dampened by properly shaping the profile, at the expense of increasing the intensity of Tollmien-Schlichting waves. The use of numerical optimization allows to perform this trade-off effectively and thus enables the extension of the NLF envelope towards the design point highlighted in the chart. The current approach follows an Infinite-Swept Wing (ISW) formulation, investigating a representative section at $70 \%$ of the wingspan. At that station, a maximum profile thickness $t / c_{\max , 0}$ of 0.11 in percentage of the chord needs to be kept to fulfil design requirements.

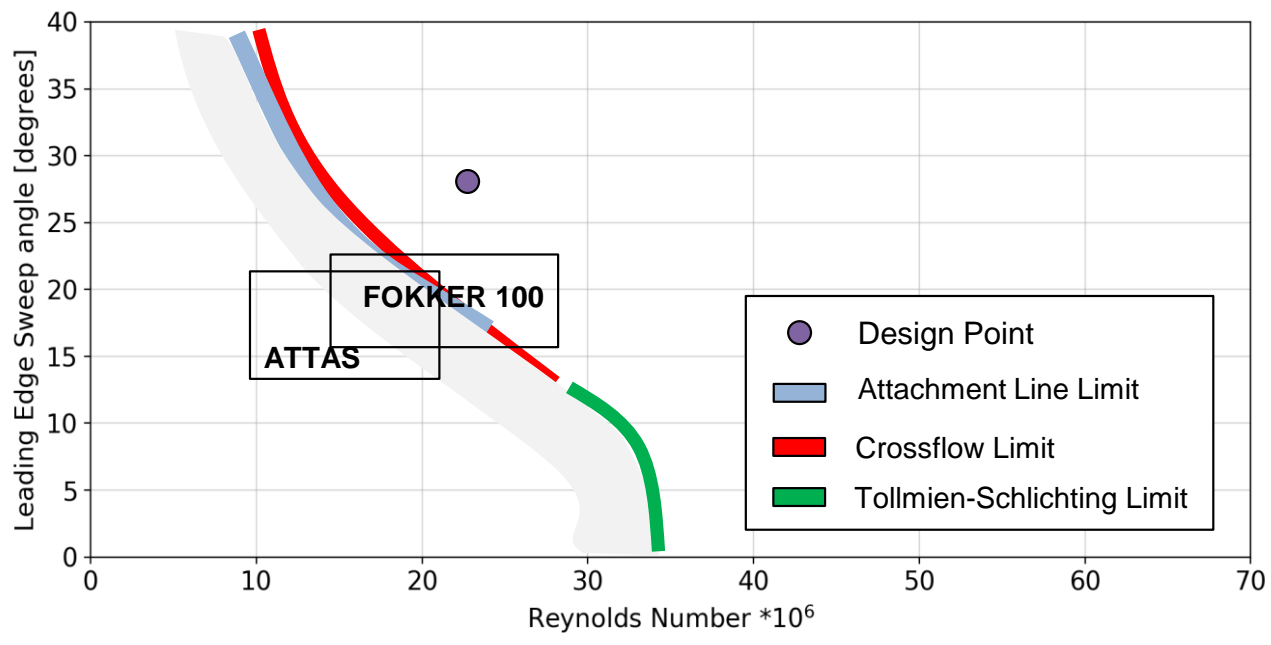

Fig. 3 Envelope of natural laminar flow suitability following previous flight tests according to [26]

\section{B. Deterministic Optimization Problem}

The deterministic global solution is defined through a classical drag optimization at constant lift coefficient, $C_{L}=0.7$, and freestream Mach number, $M=0.78$ :

$$
\begin{aligned}
\boldsymbol{x}^{*} & =\underset{\boldsymbol{x}}{\arg \min }\left\{C_{D}\left(\boldsymbol{x}, M, C_{L}\right)\right\} \\
\text { s.t. } & =\left\{\begin{array}{l}
C_{L}=C_{L_{0}} \\
t / c_{\max } \geq t / c_{\max , 0} \\
0 \leq x^{j} \leq 1, \quad j=1,2, \ldots 10
\end{array}\right.
\end{aligned}
$$

The drag coefficient $C_{D}$ depends on the ten design variables $x$ that describe the shape of the airfoil. These are dimensionless and range between zero and one. Transition prediction is enabled, with the critical N-Factors fixed 
to $N_{T S, \text { crit }}=11.5$ and $N_{C F, \text { crit }}=8.5$ [9]. The constant lift coefficient constraint is accounted for by the CFD solver directly as it iteratively changes the angle of attack while converging towards the solution to maintain the desired lift coefficient. The constraint in the maximum thickness is handled through the parametrization by rescaling the current shape in the z-direction to have the initial maximum thickness, $t / c_{\max }=0.11$.

\section{Robust Optimization Problem}

The robust solution involves both operational and environmental uncertainties accounting for realistic stochastic conditions in flight. As a result, the drag coefficient (objective function) becomes a random variable and a statistic of the drag is optimized. Two different cases are considered. On the one hand, the minimization of the mean value of the drag, $\mu_{C_{D}}$ can be seen as a natural choice from a cost-effective point of view: operators want to minimize the average fuel consumption of an aircraft over a given period of time.

$$
\boldsymbol{x}^{*}=\underset{\boldsymbol{x}}{\arg \min }\left\{\mu_{C_{D}}(\boldsymbol{x}, \boldsymbol{\xi})\right\}
$$

On the other hand, the $95 \%$ quantile, $q_{95 \%, C_{D}}$, reduces the variability of the drag by focusing on extreme cases, ensuring a minimal performance with control probability:

$$
\boldsymbol{x}^{*}=\underset{\boldsymbol{x}}{\arg \min }\left\{q_{95 \%, C_{D}}(\boldsymbol{x}, \boldsymbol{\xi})\right\}
$$

Two different configurations will arise: one with good overall performance (mean value) and another one that is better able to handle extreme events. This will give flexibility to the designer to choose the most appropriate one for the specific mission at hand.

\section{Parametrization of the Design Vector}

The shape of the profile is changed using Class Shape Function Transformations (CST) [28]. This representation captures the design space of smooth airfoils and provides smooth surfaces as $\mathrm{C} 2$ continuity is guaranteed. A total of ten design parameters are used to change the shape of the profile, five for the upper surface and five for the lower surface.

$$
\begin{gathered}
\frac{z}{c}=C\left(\frac{x}{c}\right) S\left(\frac{x}{c}\right)+\frac{x}{c} \frac{\Delta z_{T E}}{c} \\
C\left(\frac{x}{c}\right)=\left(\frac{x}{c}\right)_{1}^{N}\left(1-\frac{x}{c}\right) N_{2} \\
S\left(\frac{x}{c}\right)=\sum_{i=1}^{n_{p}} X_{i} K_{i, n}\left(\frac{x}{c}\right)^{i}\left(1-\frac{x}{c}\right)^{n-i}, \quad K_{i, n}=\frac{n !}{i !(n-1) !}
\end{gathered}
$$

\section{E. Numerical Model}

In order to obtain the aerodynamic performance, the Reynolds Average Navier Stokes (RANS) equations in conjunction with the Spalart-Allmaras turbulent model are solved by using the DLR TAU [29] code. In particular a 3v multigrid cycle, the lower/upper symmetric Gauss-Seidel implicit method for time integration in a backward Euler solver and a central flux discretization is chosen. The density residual for which simulations are seen as converged is fixed to 1e-7. The unstructured mesh is shown in Figure 4. An infinite swept-wing [30] formulation is chosen to account for crossflow effects also known as $2.5 \mathrm{D}$ analysis.

\section{F. Transition Prediction}

The transition location of 2.5D and 3D flows is predicted by local linear stability theory [31]. Despite more advanced techniques being available for the prediction of transition, this is the preferred methodology in industry [9], as this method has been validated extensively in the past through wind tunnel and flight tests. A transition prediction tool [32] is integrated within the Flowsimulator framework and consists of five elements:

1) Extraction of a slice to obtain the sectional pressure field

2) Preprocessing of the pressure field to obtain attachment line and smooth pressure contours 


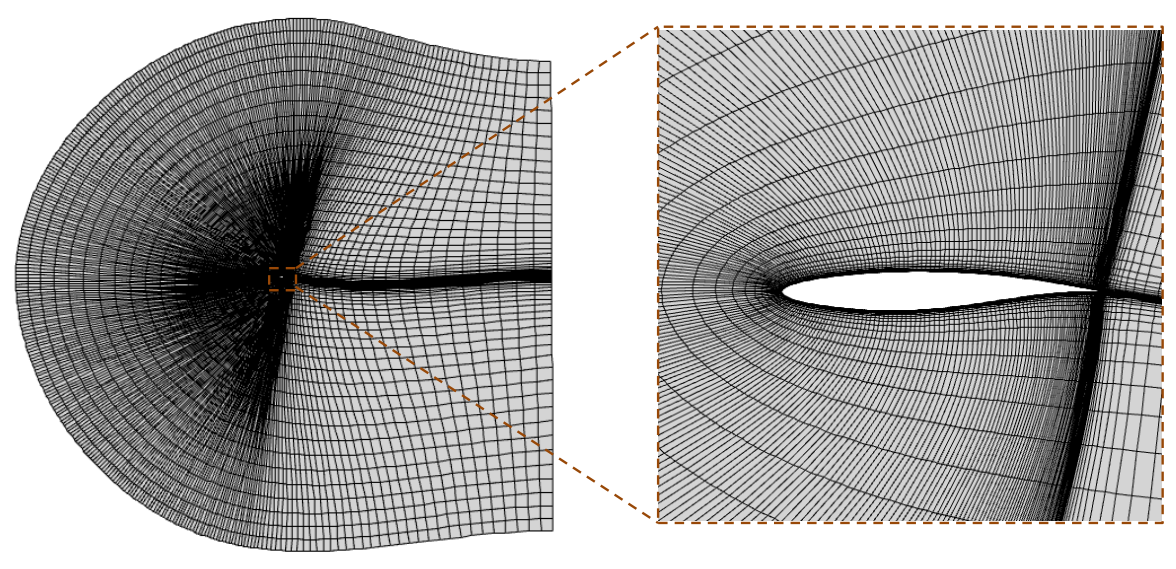

Fig. 4 CFD Grid used in the Optimization Problem

3) Use of the boundary layer solver COCO [33] (compressible conical boundary layer) to calculate the boundary layer edge velocity based on pressure contours

4) Stability analysis with LILO [32] (linear local stability analysis), using a $N_{T S}-N_{C F}$ method. $N_{T S}$ waves are obtained through a constant wave angle $\phi$ strategy covering the frequency range of unstable waves. $N_{C F}$ waves consider stationary stability with either constant wavelength $\lambda$ strategy or constant spanwise wave number $\beta^{*}$ strategy.

5) Intersection of the envelope of $\mathrm{CF}$ and $\mathrm{TS}$ waves with their respective critical N-Factors to determine the transition point.

A high-quality determination of critical N-Factors is required for an accurate prediction of the transition location. These are found according to a calibration process involving wind tunnel and flight test experiments, leading to what is called the $N_{T S}-N_{C F}$ calibration curve, as shown in Figure 5. In this case the process is the inverse to the aforementioned one. From flight tests, the transition location over the wing is measured, together with the section pressure field. The pressure field is analysed through linear stability theory. The intersection of the TS and CF envelopes with the experimental transition location marks the critical N-Factors for that experiment, which are shown as black dots in Figure 5. The Figure shows the clean data used for calibration measured during two experiments, the Fokker 100 and the ATTAS [4].

A concise presentation and discussion is given in [34]. From these data points, the $N_{T S}-N_{C F}$ calibration curve is obtained [9]. Currently, a relatively conservative approach is employed which aims for covering the envelope of the lowest critical N-Factor according to idealized experiments, as shown by the green line. In this case, the nominal critical $\mathrm{N}$-Factors (intersection of the green curve with the axis) are $N_{T S}=11.5$ and $N_{C F}=8.5$. However, in practise critical $\mathrm{N}$-Factors are highly dependent on freestream conditions such as the turbulent intensity and presence of clouds. Also, an increase in the strength of instability waves due to acoustic disturbances or manufacturing tolerances will cause a decrease in critical N-Factors. In other situations the critical N-Factor could be larger than predicted, yielding a potential that is not fully utilized. Finally, when dealing with wind tunnel testing, the critical N-Factor can change from one testing facility to another one. As a result, a probabilistic approach to the critical N-Factor is a natural extension to take these environmental uncertainties into account.

\section{G. Parametrization of Uncertainties}

To make laminar configurations more robust against situations that could cause transition more upstream than envisioned, in this work the critical $N_{T S}-N_{C F}$ calibration curve is seen as random. In practice, the calibration curve is uniquely defined by the critical N-Factors, $\left(N_{T S, c r i t}, N_{C F, c r i t}\right)$, that define the intersection of the curve with the vertical and horizontal axis respectively. In this work, these parameters are considered as two independent uncertainties that influence the whole curve. The exact probabilistic characterization of these two parameters has a direct influence on the resulting optimum configuration. In this work three different possibilities are considered, as shown in Figure 6a and Table 1

Firstly, in case A, uniform distributions $(\mathcal{U})$ are chosen for both parameters to equally weight the different possibilities 


\section{Flight Test}

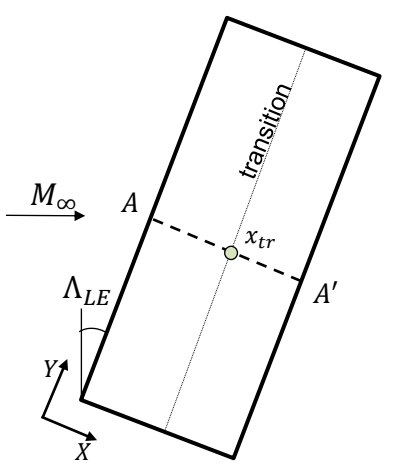

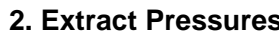

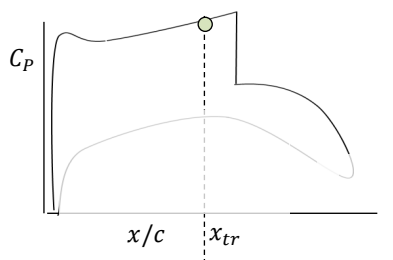

3. Run Stability Analysis Solver

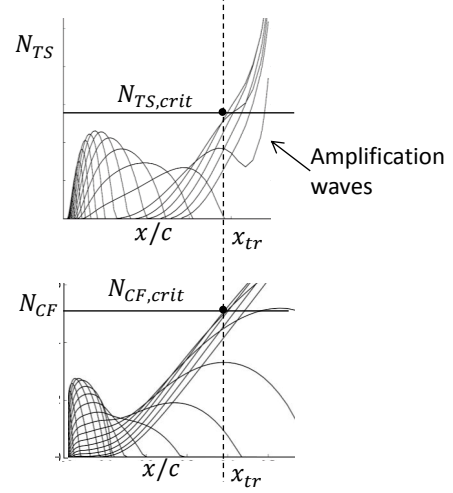

4. Record Critical N-Factor and Draw Calibration Curve

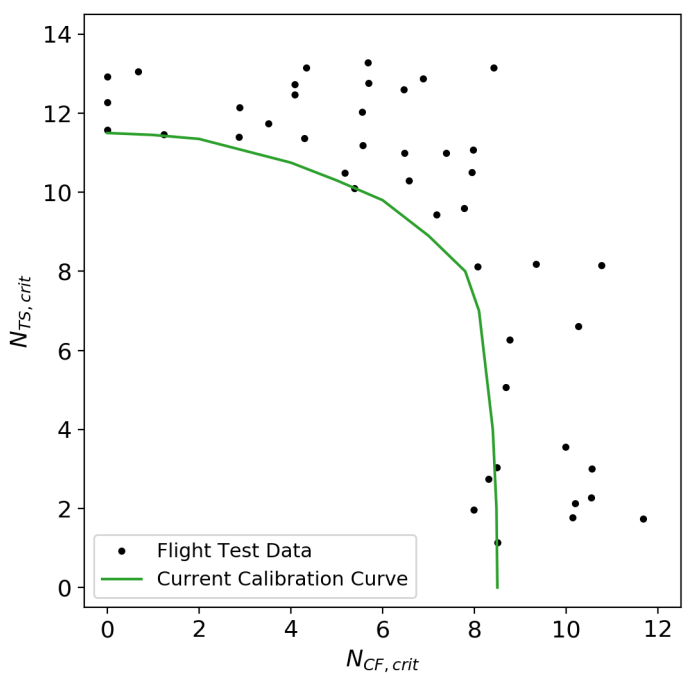

Fig. 5 Calibration process of critical N-Factor curve: measurement in flight tests / wind tunnel, extraction of pressures, evaluation of linear stability solver and recording of critical N-Factors for CF and TS waves. Flight Test data from [34], DLR calibration curve from [9]

of critical N-Factors according to a lower and upper bound. Taking into account that the calibration samples in Figure 6a were obtained under perfect conditions, it is decided to extend the feasible range to lower values to include further instabilities that could trigger transition earlier. For example, presence of clouds, ice, imperfections, bugs or simply an increase in the freestream turbulence intensity. Secondly, in case B, asymmetric Beta distributions $(\mathcal{B})$ with the parameters $r 1=20, r 2=2$ allow a more tailored weighting. In this case, the mode focuses around the central value of the calibration data, and the lower tail deals with uncommon (but feasible) scenarios. Finally, case $\mathrm{C}$ extends the critical N-Factors to zero. This conservative scenario deals with both, fully turbulent and fully laminar configurations. The choice of these probability distributions follows physical insight and is useful to test the validity of the design framework to come up with different configurations. However, these could be far from reality. In practice, more realistic probability distributions can be defined based on expert knowledge and the realization of additional flight experiments.

In addition, the Mach number and lift coefficient can be considered as operational uncertainties. These are defined according to the variability in cruise region of the short-haul configuration. In the past, these were commonly considered as fixed flight conditions in a multi-point approach. However, the framework allows to add them as additional uncertainties. They are modelled as symmetric beta distributions with $r_{1}=r_{2}=5$, centered around nominal flight conditions, as shown in Figure $6 \mathrm{~b}$.

Table 1 Possible distribution of uncertainties for critical N-Factors

\begin{tabular}{l|l|l|l} 
Case & \multicolumn{1}{|c|}{$N_{T S}$} & \multicolumn{1}{|c|}{$N_{C F}$} & \multicolumn{1}{c}{ Description } \\
\hline A & $\mathcal{U}(6,14)$ & $\mathcal{U}(4,11)$ & $\begin{array}{l}\text { Realistic approach, accounts for instabilities } \\
\text { (clouds, imperfections, freestream turbulence) }\end{array}$ \\
B & $\mathcal{B}(\mu=11.5, \sigma=1.6)$ & $\mathcal{B}(\mu=9.4, \sigma=1.6)$ & $\begin{array}{l}\text { Realistic approach, accounts for instabilities } \\
\text { with less penalization of critical cases } \\
\text { Trade-off between fully turbulent and fully laminar } \\
\text { Operating under turbulent flow }\end{array}$ \\
C & $\mathcal{U}(0,14)$ & $\mathcal{U}(0,11)$ &
\end{tabular}



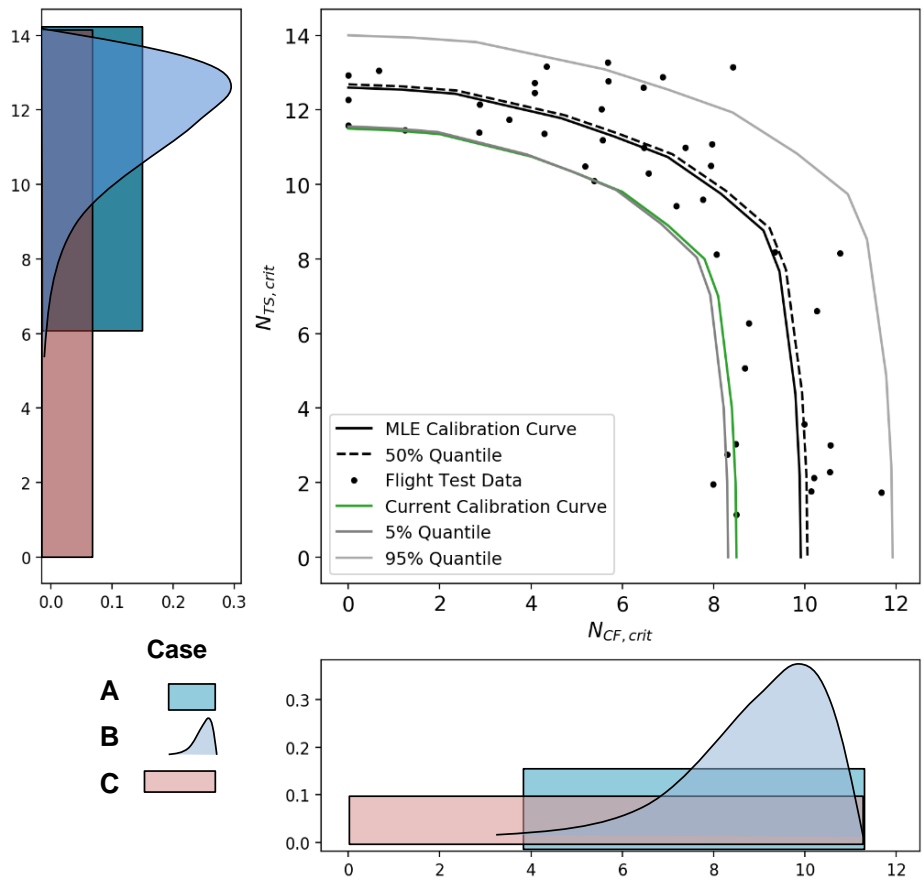

(a) Possible distributions for CF and TS critical N-Factors
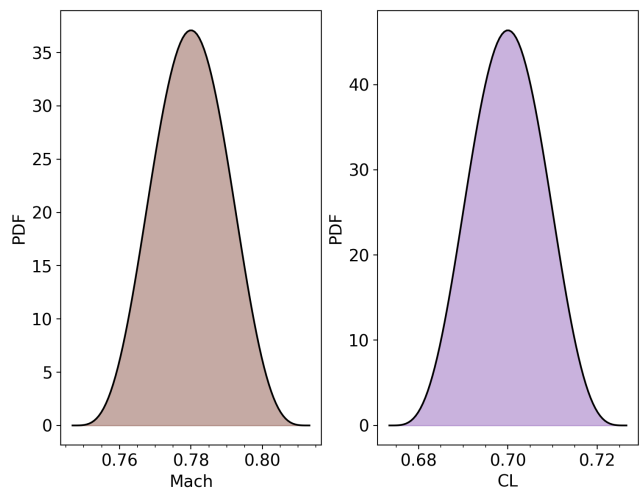

(b) Distributions for operational uncertainties: Mach number and lift Coefficient

Fig. 6 Distribution of uncertainties considered in robust design formulation

\section{H. Process Chain}

Every time the drag coefficient is required for a given design vector $\boldsymbol{x}_{\boldsymbol{i}}$, (deterministic optimization), or for a given design vector $\boldsymbol{x}_{\boldsymbol{i}}$ and uncertainties $\boldsymbol{\xi}$, (UQ in robust design), the process chain of Figure 7 is executed. The geometry is changed through a mesh deformation tool based on radial basis functions developed by DLR [35]. After the addition of the boundary conditions, the RANS equations are solved. After a preset number of iterations, the transition prediction method updates the transition location according to the given pressure distribution as outlined in section IV.F The process is repeated until convergence for the transition location and the density residual is reached. The drag coefficient is obtained by integration of pressure and viscous forces along the surface of the airfoil. The process chain relies on the Flowsimulator framework [36] and is able to handle convergence errors, restarts and parallel execution.

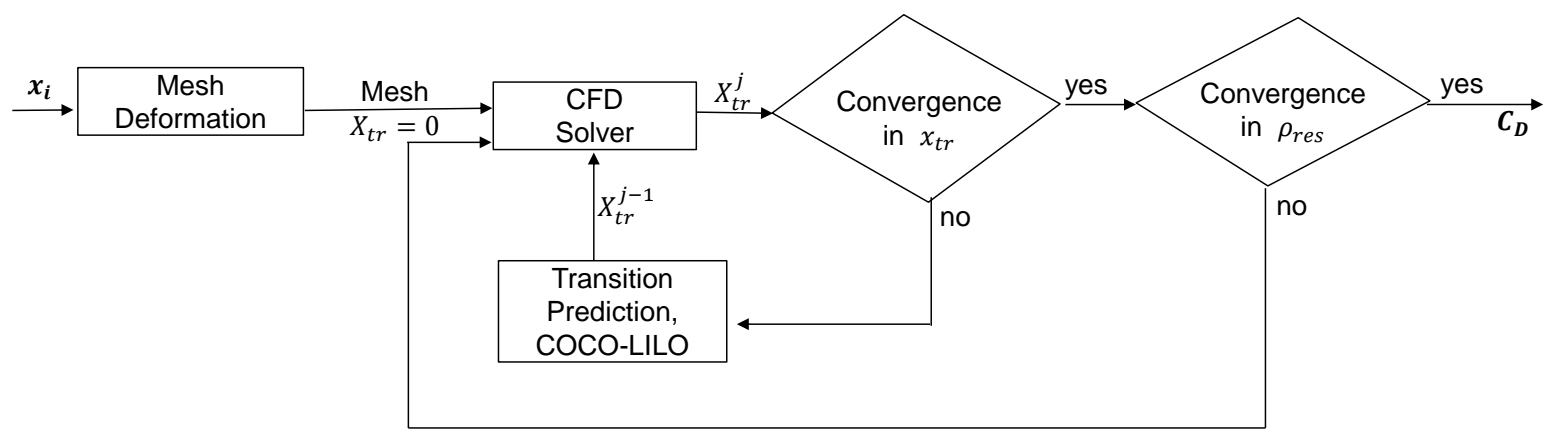

Fig. 7 CFD Process Chain to obtain the drag coefficient from a given set of design parameters 


\section{Robust Design of NLF Wings - Results}

This section presents results for deterministic and robust design of an NLF wing.

\section{A. Deterministic Optimization}

The deterministic optimization problem in equation 1 is solved with Surrogate Based Optimization. The optimization assumes the nominal critical N-Factors of $N_{T S, \text { crit }}=11.5$ and $N_{C F, \text { crit }}=8.5$. The optimum is found after 462 iterations (function evaluations), as shown in the convergence history in Figure 8 . For the ten dimensional problem, a total of 200 samples are chosen for the DoE to ensure a good initial representation of the design space. However, most of the improvement in the solution takes place during the active infill stage.

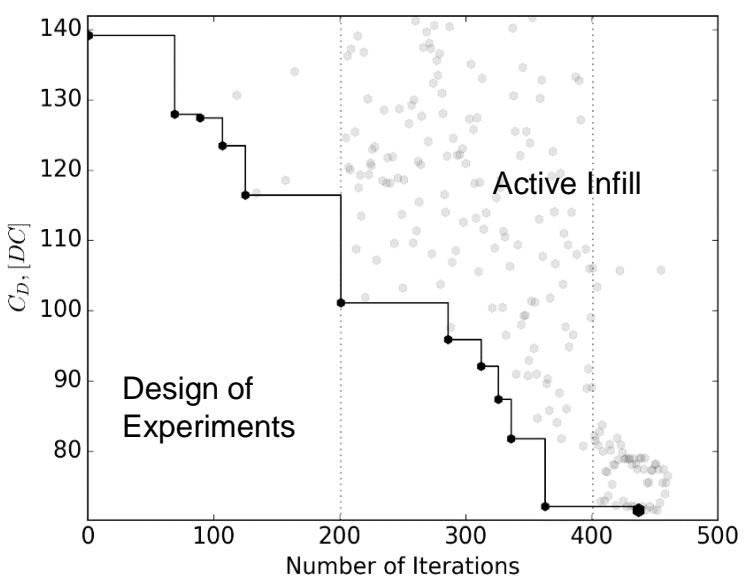

\section{Fig. 8 Convergence history of deterministic Optimization}

As shown in Figure 9, the surrogate based optimization includes the turbulent RAE2822 configuration as part of the initial DoE. The adverse pressure distribution amplifies the growth of TS waves as shown in Figure $9 \mathrm{~b}$. As a result, early transition at $10 \%$ of the chord occurs, triggered by the intersection of the critical N-Factor for TS (dotted line) with the envelope of the waves. This early transition and the strong shock wave results in a high value of drag. The optimal configuration shown in Figure 10 is able to delay transition up to the shock wave, which is roughly around $60 \%$ of the chord. The large extent of laminar flow and the weakened shock wave leads to a reduction in drag of $46.3 \%$ w.r.t. the baseline RAE2822. The obtained pressure profile is very similar to the one that would be envisioned by inverse design, and shows that the numerical optimization is able to find realistic laminar configurations only by proposing a drag minimization and enabling transition prediction.

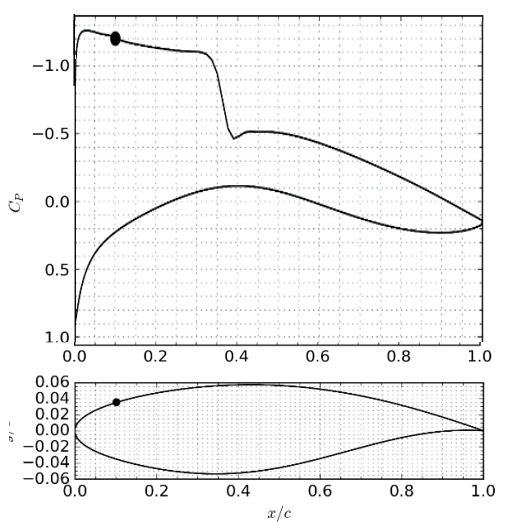

(a) Surface pressure distribution

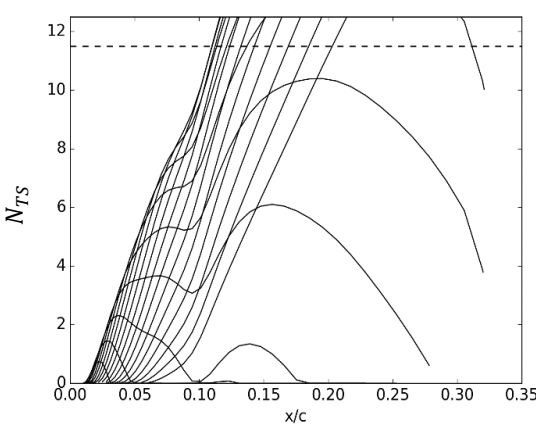

(b) Growth of TS waves

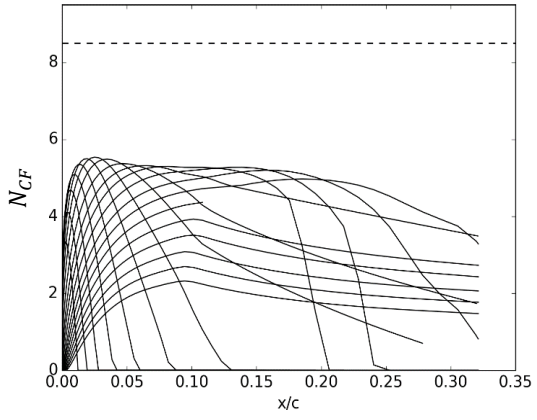

(c) Growth of CF waves

Fig. 9 Surface pressure distribution and instability waves growth for baseline configuration 


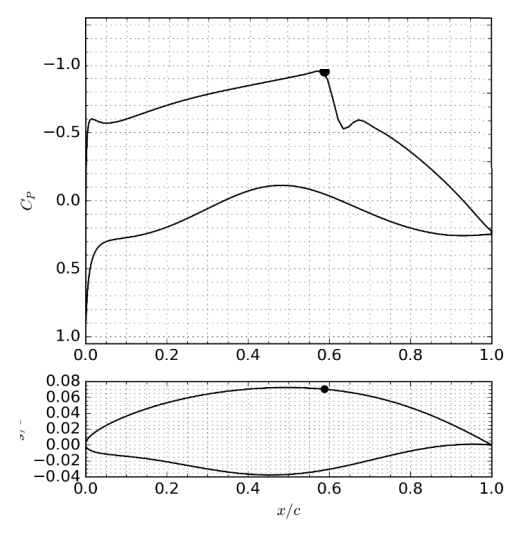

(a) Surface pressure distribution

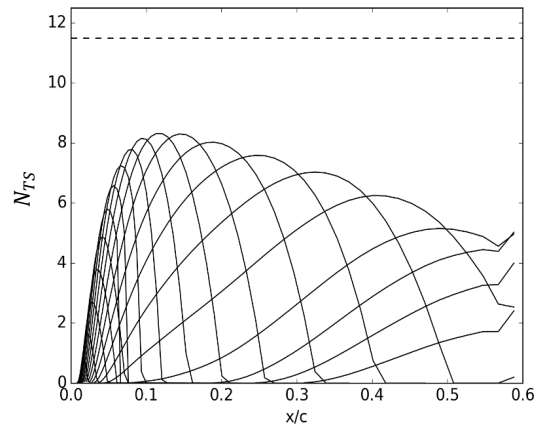

(b) Growth of TS waves

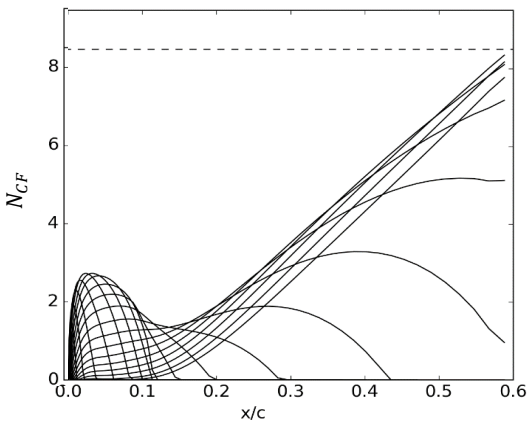

(c) Growth of CF waves

Fig. 10 Surface pressure distribution and instability waves growth for deterministic optimal configuration

Interestingly, transition occurs due to both, the shock wave and crossflow instabilities: if CF waves (Figure 10c) would be further dampened by stronger accelerations at the leading edge, there would be no change in transition location (as it can not bypass the shock location). In fact, it would instead cause an increase in drag due to a stronger shock wave. These type of trade-offs can only be effectively found with direct optimization tools. Note that, for a fair comparison, the deterministic optimal configuration is our reference solution to which robust optimization results will be compared.

\section{B. Deterministic Optimum under Uncertainties in Critical N-Factors}

The deterministic optimal configuration is sufficient as long as there is no change in critical N-Factors and in operational conditions. Looking at the development of TS waves in Figure $10 \mathrm{~b}$, a change in $N_{T S \text {, crit }}$ from 11.5 to 7 due to a change in e.g. freestream turbulence intensity would suddenly shift the transition location from $60 \%$ to approximately $5 \%$ of the chord. This behaviour is unacceptable in an industrial context, and the performance of the deterministic optimum may considerably deteriorate under realistic conditions.

The change in transition location due to the change in critical N-Factors is not straightforward and only an approximation can be obtained by following the envelope of the instability waves obtained under constant critical $\mathrm{N}$-Factors. For example, if the critical N-Factor for TS is reduced in the previous example, the transition location would be shifted, changing not only the pressure distribution but also the lift. Then, the angle of attack needs to be adjusted to retain the required lift, and the new growth of TS and CF waves would be different, leading to a different transition location. Hence only an iterative procedure will provide accurate insight and enables a consistent tracking of the phenomena. The only approach to model the sensitivity of the solution against critical N-Factors is by computing converged simulations at different critical values making use of a non-intrusive uncertainty quantification methodology.

Figures $11 \mathrm{a}$ and $11 \mathrm{~b}$ show the drag field and transition field of the deterministic optimum configuration respectively as the critical N-Factors are changing, at constant Mach and lift coefficient (no operational uncertainties present). The field is constructed based on the prediction of the Gaussian Process model which is the underlying model of the Surrogate Based Uncertainty Quantification approach as introduced in Section III.D. The ten DoE samples are represented as big black dots, while two infill samples are the green triangles which are automatically placed in the corners of the stochastic space. In these locations the surrogate error was the highest and the framework automatically selected these locations to be evaluated by the CFD to enhance the overall model accuracy. In the deterministic optimal configuration, once the critical N-Factors are reduced a sudden increase in drag and early transition due to turbulent flow is observed. As expected, there is an inverse correlation between the drag coefficient location and the transition location. This leads towards a very unstable behaviour, as a slight change in the critical N-Factors will cause a serious deterioration of the performance. 


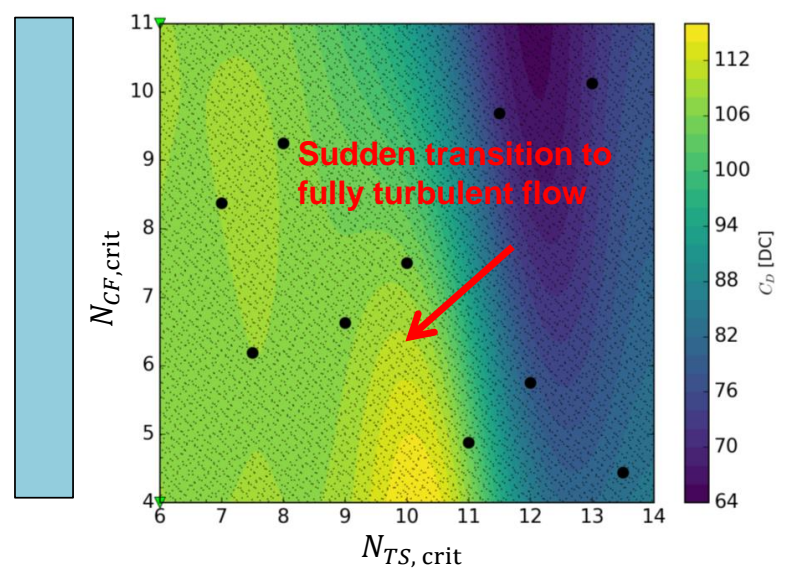

(a) Drag field, deterministic optimum, $J^{*}=C_{D}$

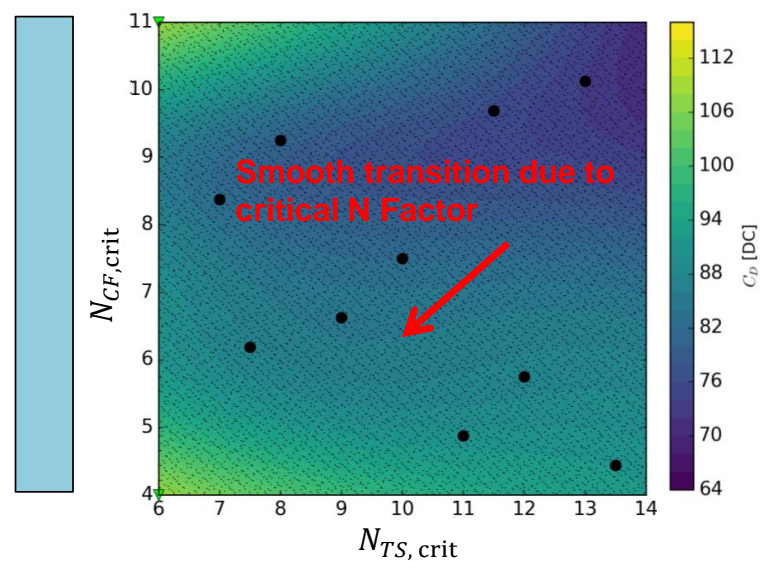

(c) Drag field, robust optimum, $J^{*}=\mu_{C_{D}}$

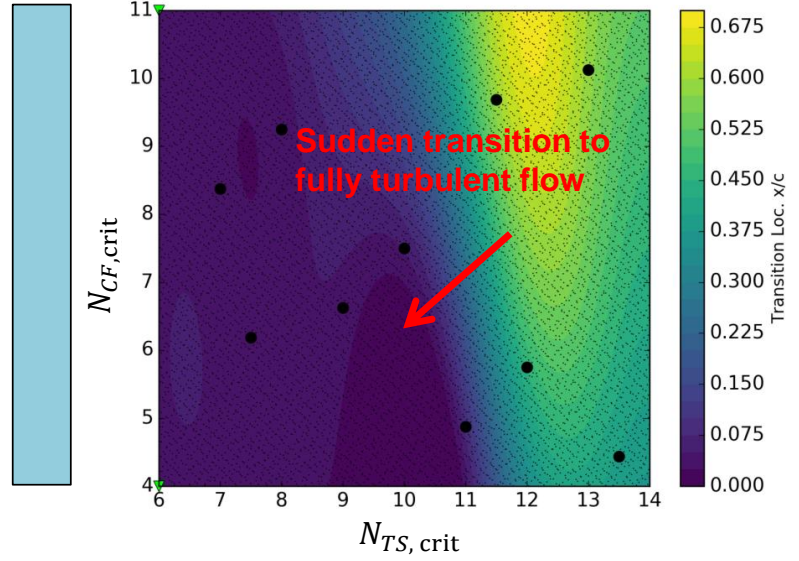

(b) Transition location field, deterministic optimum, $J^{*}=C_{D}$
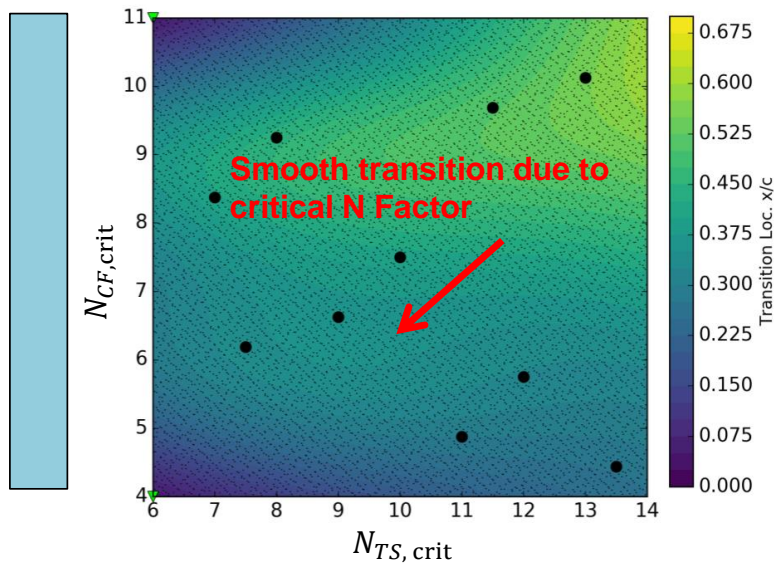

(d) Transition location field, robust optimum, $J^{*}=\mu_{C_{D}}$

Fig. 11 Drag and transition location fields predicted by the GPR model as a result of stochastic critical NFactors for Crossflow and Tollmien-Schlichting instabilities for optimal configurations. DoE points shown as big black dots, infill points represented as green triangles, Monte Carlo sampling in the surrogate as small dots

\section{Robust Optimization under Uncertainties in Critical N-Factors}

\section{Case A}

Following Table 1. Case A refers to the optimization taking uniform distributions for both $N_{T S}$ and $N_{C F}$ into account. Two different robust configurations with focus on mean and the $95 \%$ quantile respectively are obtained using the bi-level surrogate approach. The optimum which focuses on the mean of the drag coefficient, $\mu_{C_{D}}$ features a smoother correlation between changes in critical N-Factors and drag, as shown in Figure 11c. Also, following Figure 11c, laminar flow is always kept in contrast to the deterministic optima. The transition location ranges between $30 \%-55 \%$ of the chord. The resulting pressure distribution of the configuration, as shown in Figure 12a is more robust against instabilities. It dampens the TS waves shown in Figure $12 \mathrm{~b}$, reducing the peak from $N_{T S}=8.5$ to $N_{T S}=4.5$ while retaining a good performance over all possible N-Factors compared to the deterministic solution. This makes the configuration more robust. A similar behaviour occurs when looking at the robust optimum in the $95 \%$ quantile, $q_{95 \%, C_{D}}$. As shown in Figure 13a, the normal shock wave is replaced by two weaker isentropic shock waves, reducing wave drag especially when fully turbulent flow is present. As a result, the drag in the region with the "worst" performance (turbulent flow) is reduced, as expected by the $95 \%$ quantile formulation. In addition, the growth of TS and CF waves is also dampened, so 


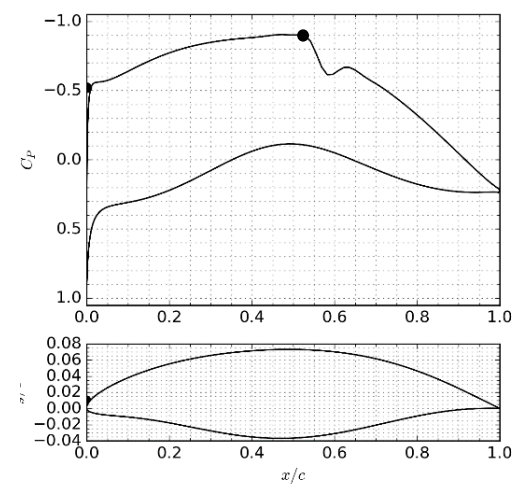

(a) Surface pressure distribution

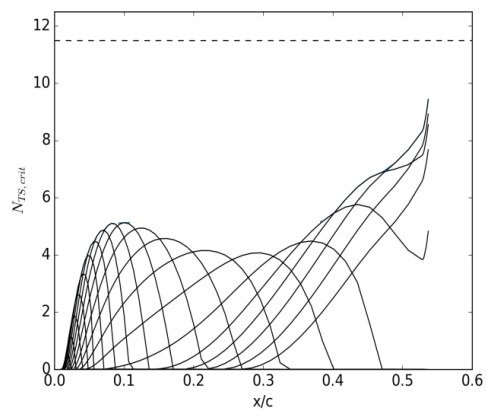

(b) Growth of TS waves

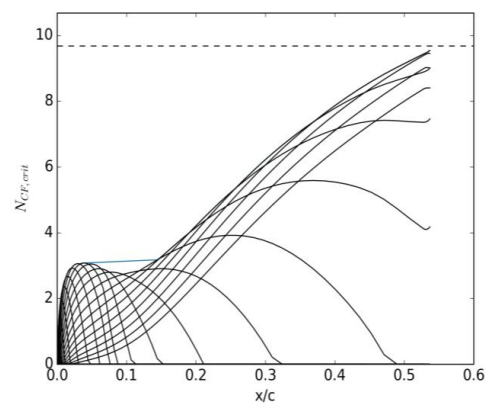

(c) Growth of CF waves

Fig. 12 Surface pressure distribution and instability waves growth for robust optimum $\mu_{C_{D}}$ configuration under case A

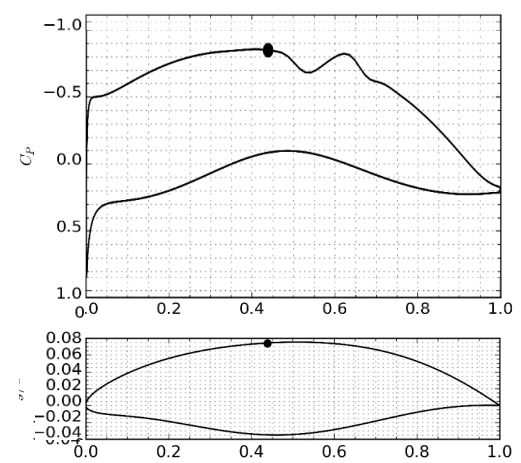

(a) Surface pressure distribution

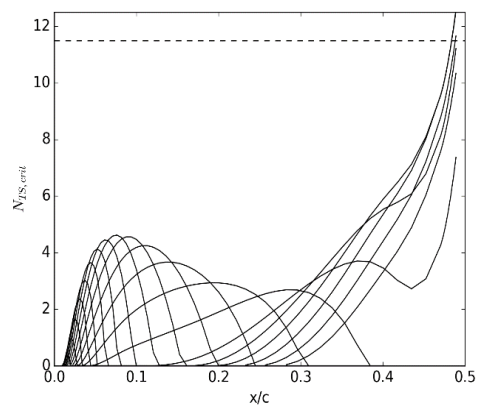

(b) Growth of TS waves

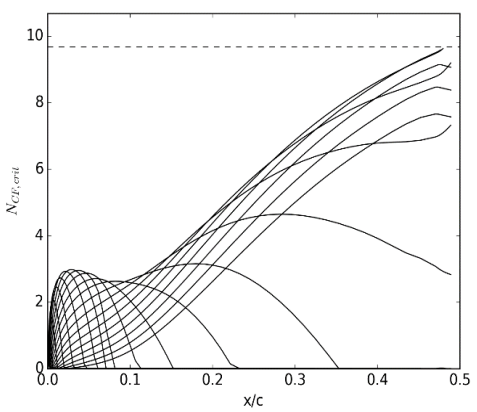

(c) Growth of CF waves

Fig. 13 Surface pressure distribution and instability waves growth for robust optimal $q_{95 \%, C_{D}}$ configuration under case A

the laminar flow performance is also improved, but not as significant as with the optimum in the mean value.

The stochastic performance of the different configurations is summarized in the violin plots in Figure 14. The probability density functions of the drag coefficients are mirrored along the vertical axis for the three different optimums. The average value as well as the quantiles are shown in the box-and-whisker plots on top of the probability density functions. As expected, the deterministic optimum (blue) features the configuration with the highest mean drag. The observed peak of the PDF towards 110 drag counts is caused by fully turbulent flow. Even though under fully laminar flow this configuration performs very good (represented by the lower tail), it is very brittle at higher critical N-Factors, leading towards a worst performance. This problem is successfully leveraged by the robust optimum with focus on the mean $\mu_{C D}$ (orange). This configuration has the lowest average, and shifts the distribution of drag towards lower values. Even though under fully laminar flow (ideal conditions) it does not perform as good as the deterministic optima, in practice (changing conditions) it will feature a better performance. Finally, the optimum configuration which focuses on the $95 \%$ quantile (green) reduces the upper tail of the distribution. As previously explained this primarily stems from a better turbulent performance due to the aforementioned double shock wave.

Ten random realizations of the pressure and transition locations are shown in Figure 15 for each optimal configuration. It clearly outlines the change in pressure and transition location in case of uncertain critical N-Factors. On the one hand, the deterministic optimum, Figure 15a, will be operating under fully turbulent flow a considerable amount of time. The upstream movement of the shock wave can be seen as transition occurring earlier. On the other hand, in Figures $15 \mathrm{~b}$ and $15 \mathrm{~b}$ both robust optima have a more gentle variation of the transition location under random changes of the N-Factors, 
reducing the longitudinal movement of the shock wave.

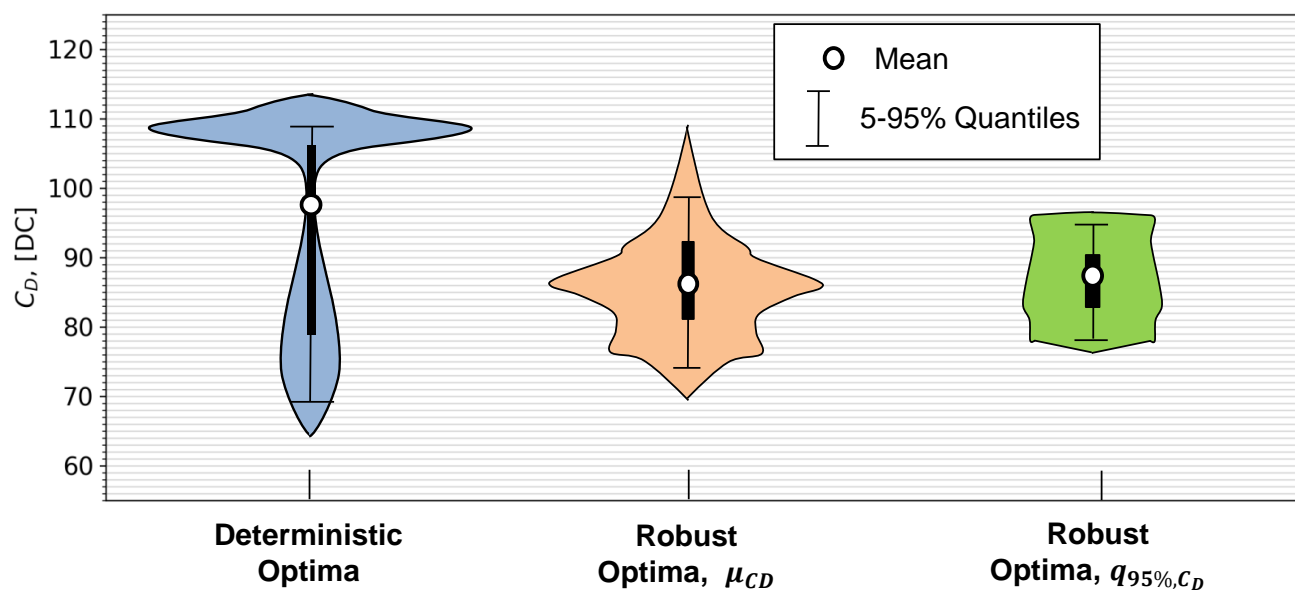

Fig. 14 Violin Plot of optimal configurations under environmental uncertainties, case A

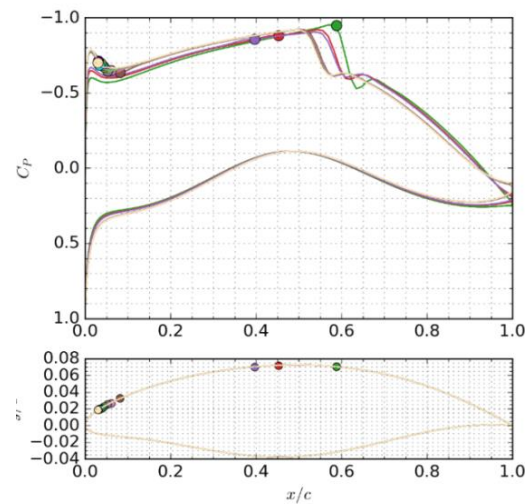

(a) Deterministic Optimum

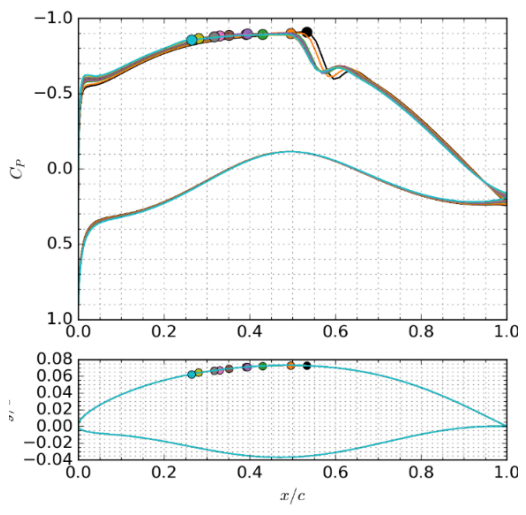

(b) Robust Optimum, $\mu_{C_{D}}$

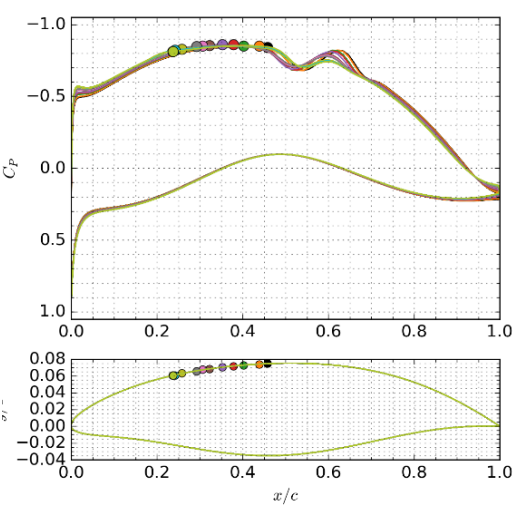

(c) Robust Optimum, $q_{95 \%}, C_{D}$

Fig. 15 Random realizations of surface pressure coefficient and transition location under environmental uncertainties, case A

A deeper insight can be provided from the TS-CF growth plots shown in Figure 16 The black line represents the growth of the N-Factors along the chord of the wing, shown by the red numbers. As the flow propagates downstream along the chord, instabilities arise and both N-Factors increase. The critical N-Factor envelope is shown in dotted blue for a given set of critical $N_{T S}$ and $N_{C F}$. When the black line intersects with the envelope, instabilities reach their critical values and transition to turbulent flow is triggered. The deterministic optimum, shown in Figure 16a, inhibits a very favourable pressure distribution for laminarity. The black line never reaches the envelope. In that case transition occurs due to the presence of the shock wave. However this makes it very unstable against changes in the critical N-Factors which define the blue envelope. It is stochastic and changes according to the environmental conditions. As the N-Factor growth is parallel to the envelope, if this one reduces, transition is deteriorated instantly along the whole chord. The TS-CF growth of the robust configurations crosses the envelop perpendicularly. This leads to a more gradual and less sudden decrease in the transition location in case of a reduction in the envelope. This phenomena, known to experts in the field, was not considered explicitly by the optimizer. By solely setting up the robust optimization formulation, the optimizer was able to come up with configurations which feature these desirable properties. 


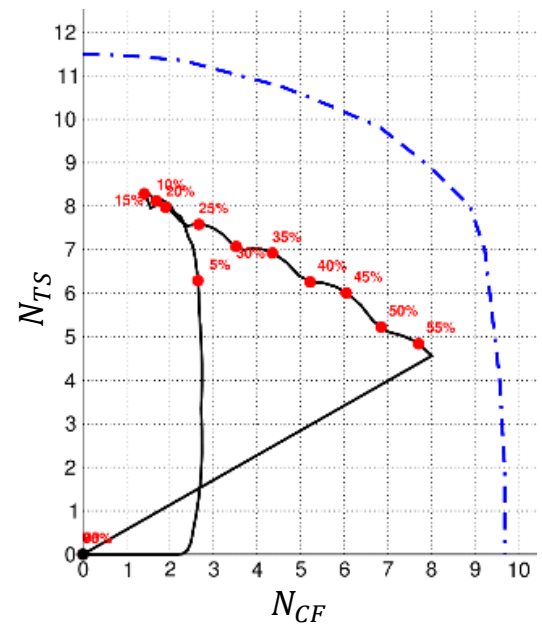

(a) Deterministic Optimum

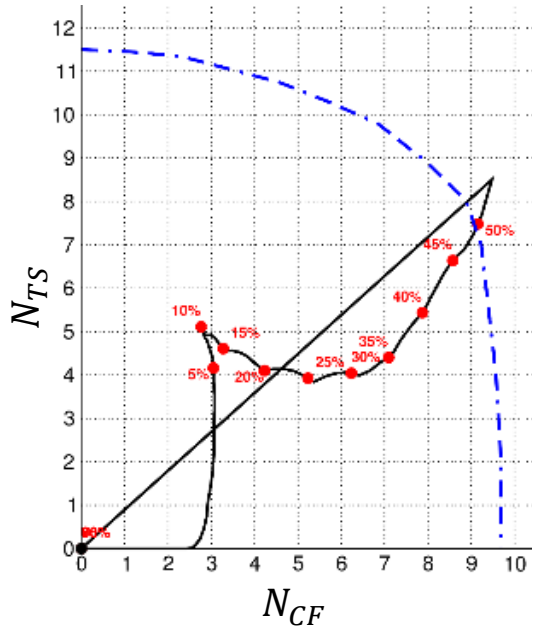

(b) Robust Optimum, $\mu_{C_{D}}$

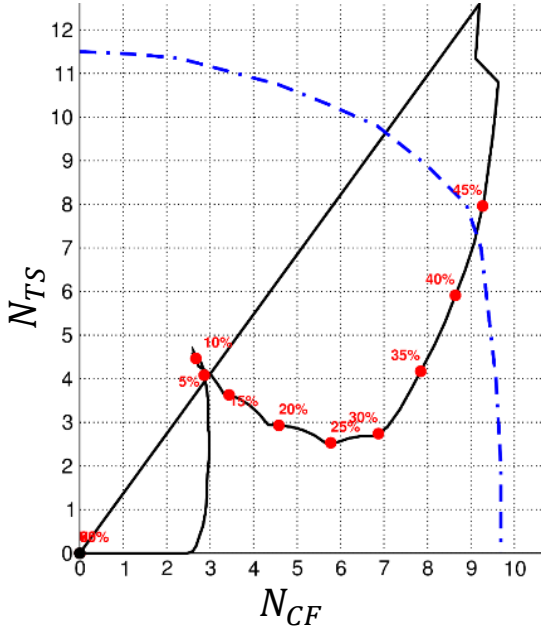

(c) Robust Optima, $q_{95 \%, C_{D}}$

Fig. $16 N_{T S}$ and $N_{C F}$ Factor growth (black line) and percentage of the chord that reaches reaches that given N-Factor (red numbers) for different configurations. Critical N-Factor envelope (blue-dotted line) is also represented. When the $\mathrm{N}$-Factor growth crosses the envelope, instabilities in the boundary layer trigger transition

\section{Case B}

Case B takes into account a different representation of the uncertainties in the critical N-Factors by considering beta distributions. In this case, higher critical N-Factors are favoured and extreme cases (lower critical N-Factor) are more rare following the shape of the PDF in Figure 6a Looking at the Violin plots in Figure 17, the peak of the distributions (maximum likelihood) lean towards the lowest values of drag. This means that most of the time these configurations will perform as expected and laminar flow over the upper surface is present. Still, due to the finite (but small) probability of lower critical N-Factors (instabilities that could prematurely trigger transition), the deterministic optimum PDF presents a relatively long tail towards higher values of drag. This is solved with both robust configurations. They present the best compromise between good laminar performance and off-design performance due to environmental uncertainties.

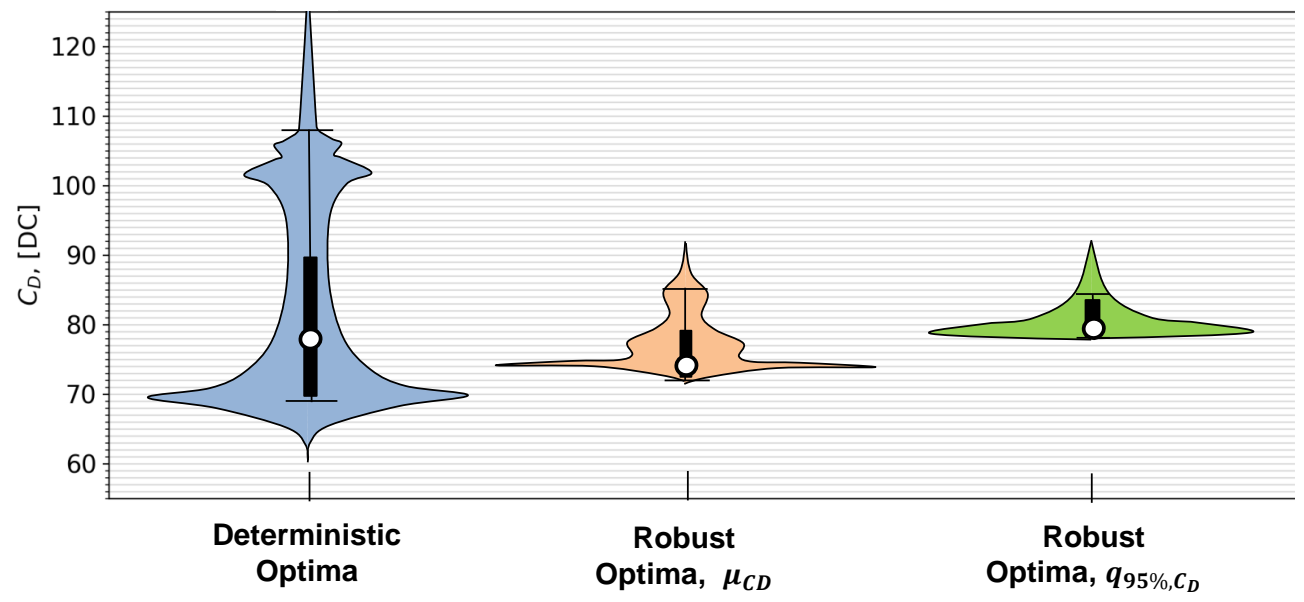

Fig. 17 Violin Plot of optimal configurations under environmental uncertainties, case B

The good compromise in behaviour is also shown in Figure 18 by selecting ten random realizations of the pressure coefficient. Note the range of the transition location for the different configurations: between $0 \%$ and $60 \%$ of the chord for the deterministic optimum in Figure 18a, between 30\% and 52\% for the optimum in mean in Figure $18 \mathrm{~b}$ and between 
$35 \%$ to $46 \%$ of the chord for the optimum $95 \%$ quantile in Figure $18 \mathrm{c}$ Due to the reduced tail in drag coefficient caused by the small range of transition locations, the latter configuration could be a good candidate for a "conservative" design for which it is important to guarantee a good performance $95 \%$ of the time.

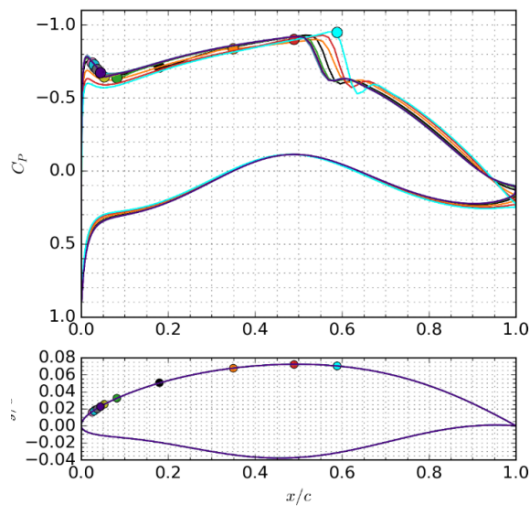

(a) Deterministic Optimum

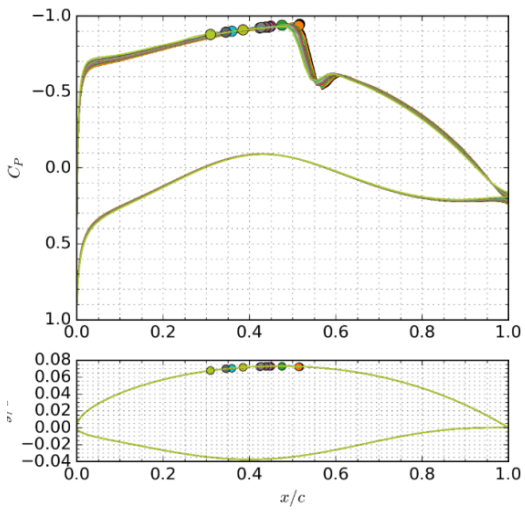

(b) Robust Optimum, $\mu_{C_{D}}$

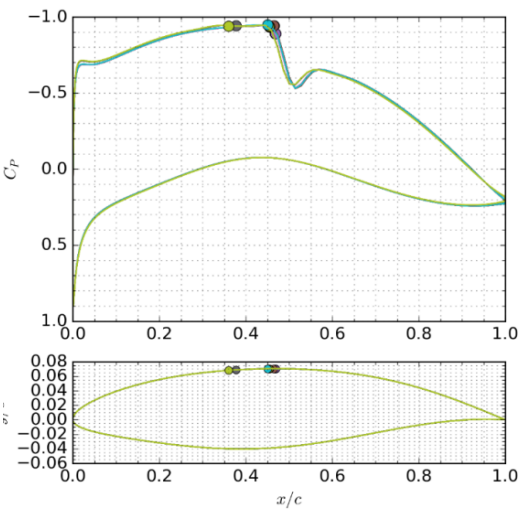

(c) Robust Optimum, $q_{95 \%}, C_{D}$

Fig. 18 Random realizations of surface pressure coefficient and transition location under environmental uncertainties, case B

\section{Case $C$}

Case $\mathrm{C}$ extends the uniform distributions of the critical N-Factors until 0. It is a conservative design approach to deal with both fully turbulent and laminar configurations by giving the same importance to both cases. The PDFs of the three configurations (deterministic, optimum in mean and optimum in $95 \%$ quantile) are shown in the violin plot of Figure 19 In contrast to the previous cases, the peak of the PDFs are shifted towards higher values of drag, as this time, fully turbulent flow is predominant due to the larger extent of critical $\mathrm{N}$-Factor also including 0 . The robust optimizer chooses to minimize the drag under fully turbulent conditions. The price to pay is a smaller laminar region and earlier transition compared to the deterministic optimum. As a result, the PDF for the robust configurations is shifted down.

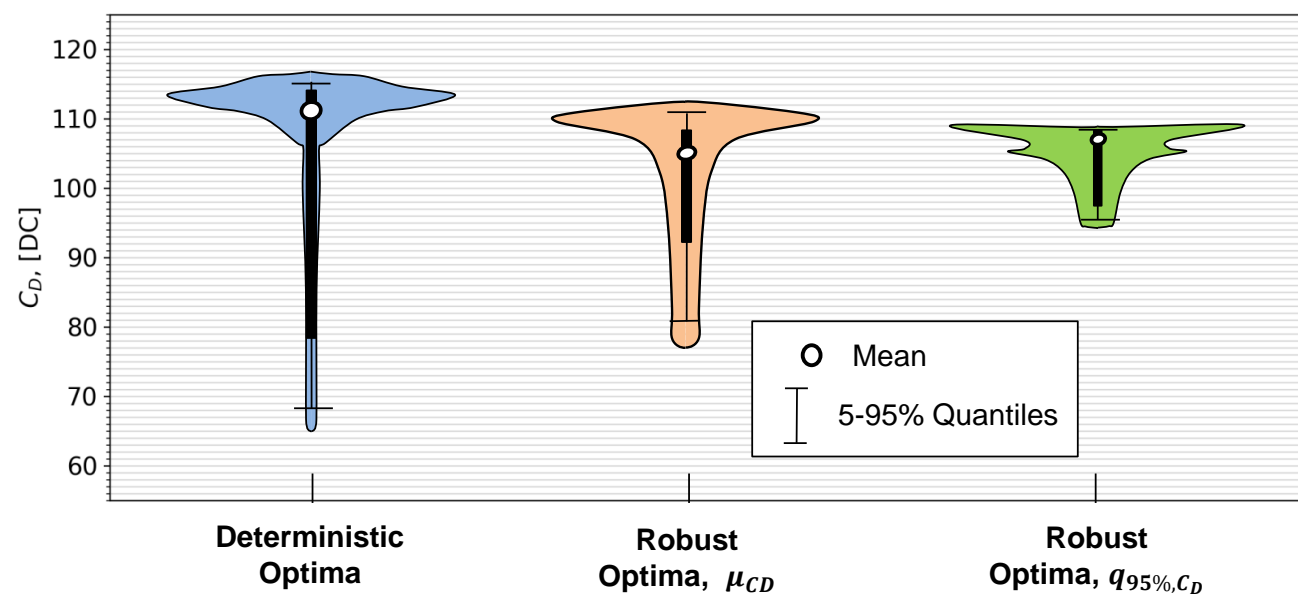

Fig. 19 Violin Plot of optimal configurations under environmental uncertainties, case C

In Figure 20 random realizations of the pressure are selected together with the transition location for these three shapes. The robust configurations decrease the shock wave strength under fully turbulent conditions. There is a decreased interest in maintaining a favourable pressure gradient to delay instabilities, as turbulent flow is predominant, and this would only lead to increased wave drag. This phenomena is of particular importance for the optimization of the 95\% quantile of the drag in Figure 20c In that case, the reduction in the shock strength is penalized by a decrease in the 
slope of the pressure coefficient, leading to a conservative configuration with a smaller laminar extent.

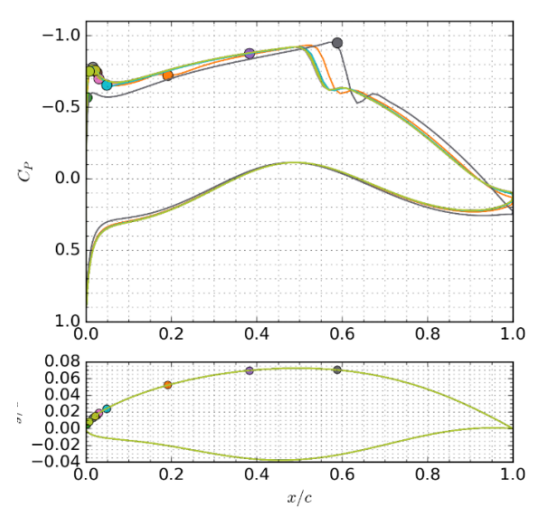

(a) Deterministic Optimum

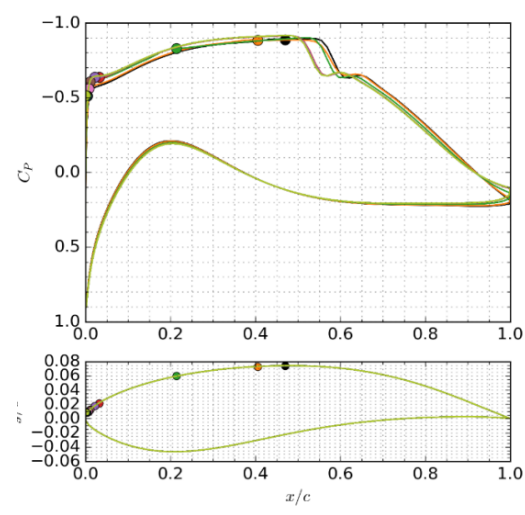

(b) Robust Optimum, $\mu_{C_{D}}$
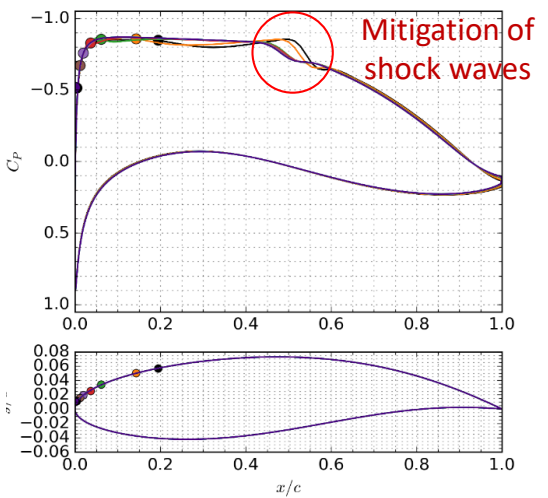

(c) Robust Optimum, $q_{95 \%}, C_{D}$

Fig. 20 Random realizations of surface pressure coefficient and transition location under environmental uncertainties, case $\mathrm{C}$

\section{Robust Optimization under Uncertainties in Critical N-Factors and Operational Conditions}

In the final application, operational uncertainties in Mach number and lift coefficient are added to the already investigated environmental uncertainties in critical N-Factors following case A. The addition of these uncertainties increases the number of samples required for the surrogate based uncertainty quantification. Figure 21 shows the violin plots for three configurations. The deterministic optima, in blue, shows a large average and variability in performance. The robust optima previously obtained taking only uncertainties in the critical N-Factor (case A) into account is shown in orange. Even though Mach and lift variations are not accounted for directly when designing this configuration, it features an acceptable behaviour with reduced average drag and a downshift of the distribution. This shows that robustness against operating conditions is indirectly taken into account by accounting for the variability in the critical $\mathrm{N}$-Factors. Finally, the new configuration taking into account uncertainties in critical N-Factor, Mach and lift coefficient is shown in red. As expected, it shows the best overall performance by not only decreasing drag but also lowering the tail.

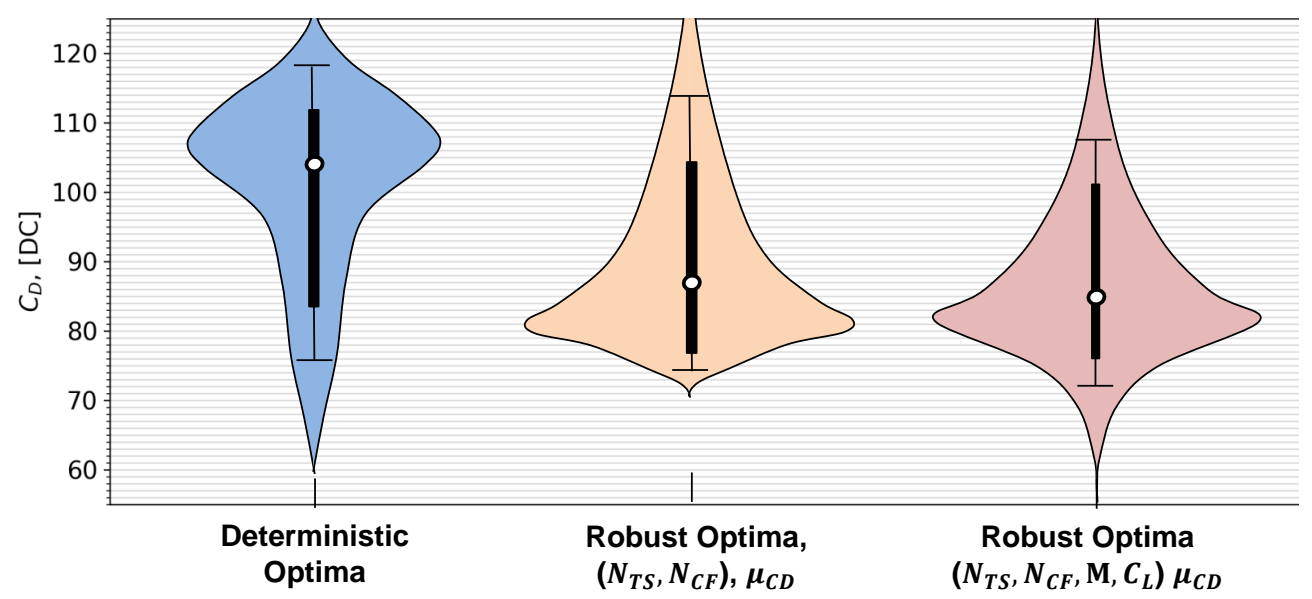

Fig. 21 Violin Plot of optimal configurations under environmental (case A) and operational uncertainties

Looking at the realizations of the pressure coefficients in Figure 22, a larger variability in the pressure due to the changes in lift coefficient and Mach number is present. Still, the robust configuration in Figure 22c, corresponding to the red PDF in Figure 21 is able to always keep a laminar flow over the upper surface. 

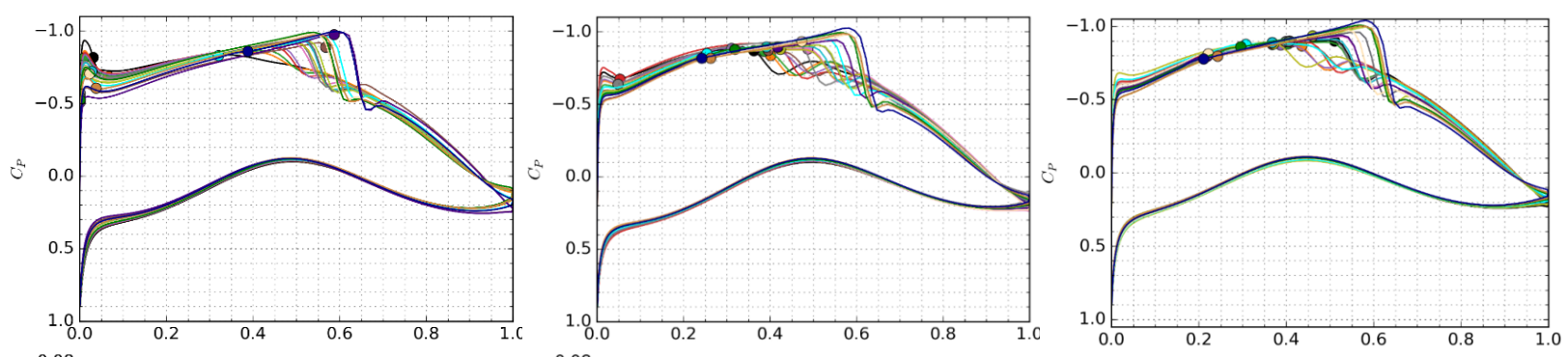

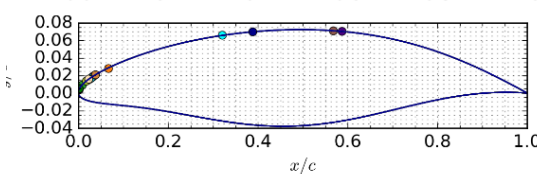

(a) Deterministic Optimum

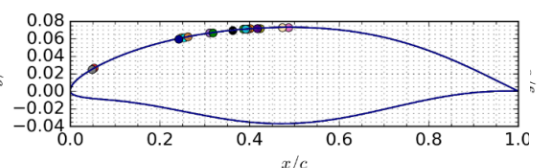

(b) Robust Optimum, $\left(N_{T S}, N_{C F}\right) \mu_{C_{D}}$

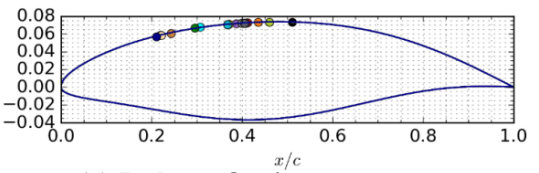

(c) Robust Optimum, $\mu_{C_{D}}$

Fig. 22 Random realizations of surface pressure coefficient and transition location under environmental uncertainties, case $\mathbf{C}$

Finally, the polar at constant weight is used to compare the performance of the different configurations in Figure 23. The continuous lines show the drag coefficient as a function of the Mach number keeping the weight constant. The lift coefficient is then changed in order to keep the product $C_{L} M^{2}=K$ constant, where $K$ is a constant obtained at cruise conditions: $C_{L}=0.7$ and $M=0.78$. The polars are drawn at the nominal critical N-Factors of $N_{T S}=11.5$ and $N_{C F}=8.5$. In all three cases, the drag bucket is shown. As expected, the deterministic optimum (blue line), has the lowest drag at the design point, but has also the smallest drag bucket extension. There is less freedom regarding the flight speed aircraft should be operating in order to benefit from the optimization potential. Both robust optimum (orange and red lines) have an extended drag bucket and as a result, are more robust against changes in Mach number. In addition, the robust optimum taking into account both environmental and operational uncertainties has the lowest drag around $M=0.77$. In addition, the polar of the robust optima under fully turbulent flow is also shown. For comparison, the transition location is shown for each Mach number as dotted lines.

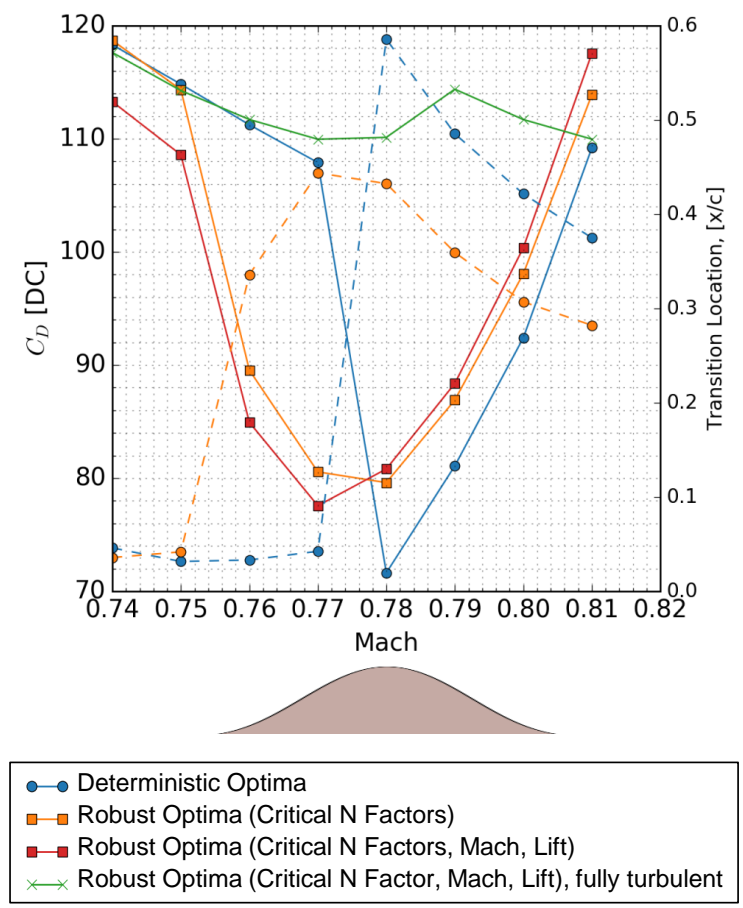

Fig. 23 Polar at constant weight for the different configurations 


\section{Conclusions}

The robust design of laminar flow wings is critical to come up with shapes that are able to retain laminarity while environmental and operational uncertainties are present. Otherwise, the theoretical improvements in fuel consumption that laminar configurations offer will be lost in practice. Current inverse design approaches for NLF design not only tend to come up with suboptimal configurations, but also face severe challenges regarding the incorporation of robustness against changes in environmental and operational conditions. The use of direct (deterministic) design optimization techniques result in realistic pressure distributions that are able to delay transition until the shock and minimize drag at nominal conditions. However, these shapes can quickly become fully turbulent if flying under a different critical $\mathrm{N}$-Factor than the one used to predict transition during the optimization. The use of a probabilistic approach for the design of natural laminar flow airfoils and wings enables new configurations that are more resilient to changes in environmental and operational conditions.

The first step to achieve robustness is the development of an efficient framework for the optimization under uncertainty of natural laminar flow wings. Environmental conditions can be modelled as uncertainties in the critical N-Factors for Tollmien-Schlichting and Crossflow instability waves, making the $N_{T S}-N_{C F}$ critical envelope stochastic. Operational conditions can be accounted for through uncertainties in Mach number and lift coefficient. The combination of state-of-the-art tools such as a computational fluid dynamics solver, linear stability theory and surrogate models allow to efficiently obtain robust shapes within a feasible computational budget. In the robust design case, the objective function is shifted from the drag coefficient towards a statistic of the drag, such as the mean value to minimize average fuel consumption over a period of time, or the $95 \%$ quantile to minimize extreme events ensuring minimal performance losses with controlled probability.

The robust design framework has been applied to a natural laminar flow infinite swept wing for a short-haul aircraft configuration, extending the current design envelope for natural laminar flow design. Initially it is demonstrated that the deterministic optimum is, as stated, not robust against instabilities, due to the growth of Tollmien-Schlichting waves close to its critical N-Factor. By considering appropriate probability distributions, it is possible to enhance the average performance or the $95 \%$ quantile of the drag, reducing the impact of extreme events that could suddenly trigger transition. The N-Factor growth of the robust shapes crosses perpendicularly the $N_{T S}-N_{C F}$ critical envelope. As a result, these feature a smooth decrease in transition location with the increase of instabilities, instead of a sudden change that triggers fully turbulent flow. The exact behaviour can be tailored according to the probability distributions of the critical N-Factors. The addition of operational uncertainties to the optimization problem increases the extent of the drag bucket, increasing the operating range of NLF wings. In the future, more realistic distributions for the critical N-Factors can be developed according to expert knowledge or additional flight tests.

\section{Acknowledgments}

The authors would like to thank the Component Aerodynamics Team in Airbus UK for their feedback and support during the development of this work. This work is funded by the European Commission's H2020 programme, through the UTOPIAE Marie Curie Innovative Training Network, H2020-MSCA-ITN-2016, Grant Agreement number 722734.

\section{References}

[1] Green, J. E., "Civil aviation and the environment - the next frontier for the aerodynamicist," The Aeronautical Journal, Vol. 110, No. 1110, 2006, pp. 469-486. https://doi.org/10.1017/s0001924000001378.

[2] Beck, N., Landa, T., Seitz, A., Boermans, L., Liu, Y., and Radespiel, R., “Drag Reduction by Laminar Flow Control,” Energies, Vol. 11, 2018, p. 252. https://doi.org/10.3390/en11010252.

[3] Green, J., "Laminar Flow Control - Back to the Future?" 38th Fluid Dynamics Conference and Exhibit, American Institute of Aeronautics and Astronautics, 2008. https://doi.org/10.2514/6.2008-3738

[4] REDEKER, G., HORSTMANN, K., KOESTER, H., THIEDE, P., and SZODRUCH, J., "Design for a natural laminar flow glove for a transport aircraft," Flight Simulation Technologies Conference and Exhibit, American Institute of Aeronautics and Astronautics, 1990. https://doi.org/10.2514/6.1990-3043.

[5] McLean, D., Understanding aerodynamics: arguing from the real physics, Wiley, Chichester, West Sussex, United Kingdom, 2013.

[6] Schrauf, G., "EVALUATION OF THE A320 HYBRID LAMINAR FIN EXPERIMENT," European Congress on Computational Methods in Applied Sciences and Engineering, ECCOMAS: Barcelona, Spain, 2000. 
[7] Campbell, R. L., and Lynde, M. N., "Natural Laminar Flow Design for Wings with Moderate Sweep," 34th AIAA Applied Aerodynamics Conference, American Institute of Aeronautics and Astronautics, 2016. https://doi.org/10.2514/6.2016-4326

[8] Lynde, M. N., Campbell, R. L., Rivers, M. B., Viken, S. A., Chan, D. T., Watkins, A. N., and Goodliff, S. L., "Preliminary Results from an Experimental Assessment of a Natural Laminar Flow Design Method," AIAA Scitech 2019 Forum, American Institute of Aeronautics and Astronautics, 2019. https://doi.org/10.2514/6.2019-2298, URL https://doi.org/10.2514/6.2019-2298.

[9] Streit, T., Wedler, S., and Kruse, M., "DLR natural and hybrid transonic laminar wing design incorporating new methodologies," The Aeronautical Journal, Vol. 119, No. 1221, 2015, pp. 1303-1326. https://doi.org/10.1017/s0001924000011283

[10] Crouch, J., Sutanto, M., Witkowski, D., Watkins, A., Rivers, M., and Campbell, R., "Assessment of the National Transonic Facility for Natural Laminar Flow Testing," 48th AIAA Aerospace Sciences Meeting Including the New Horizons Forum and Aerospace Exposition, American Institute of Aeronautics and Astronautics, 2010. https://doi.org/10.2514/6.2010-1302. URL https://doi.org/10.2514/6.2010-1302

[11] Skinner, S., and Zare-Behtash, H., "State-of-the-art in aerodynamic shape optimisation methods," Applied Soft Computing, Vol. 62, 2018, pp. 933-962. https://doi.org/10.1016/j.asoc.2017.09.030

[12] Han, Z.-H., Chen, J., Zhang, K.-S., Xu, Z.-M., Zhu, Z., and Song, W.-P., "Aerodynamic Shape Optimization of NaturalLaminar-Flow Wing Using Surrogate-Based Approach,” AIAA Journal, Vol. 56, No. 7, 2018, pp. 2579-2593. https: //doi.org/10.2514/1.j056661, URL https://doi.org/10.2514/1.j056661

[13] Zhao, H., Gao, Z., Wang, C., and Yuan, G., "Robust Design of High Speed Natural-Laminar-Flow Airfoil for High Lift," 55th AIAA Aerospace Sciences Meeting, American Institute of Aeronautics and Astronautics, 2017. https://doi.org/10.2514/6.2017-1414. URL https://doi.org/10.2514/6.2017-1414

[14] Quagliarella, D., and Iuliano, E., "Robust Design of a Supersonic Natural Laminar Flow Wing-Body," IEEE Computational Intelligence Magazine, Vol. 12, No. 4, 2017, pp. 14-27.

[15] Hollom, J., and Qin, N., "Robustness of Natural Laminar Flow Airfoil Drag Optimization to Transition Amplification Factor," 18th AIAA/ISSMO Multidisciplinary Analysis and Optimization Conference, American Institute of Aeronautics and Astronautics, 2017. https://doi.org/10.2514/6.2017-3144. URL https://doi.org/10.2514/6.2017-3144.

[16] Schuëller, G., and Jensen, H., "Computational methods in optimization considering uncertainties - An overview," Computer Methods in Applied Mechanics and Engineering, Vol. 198, No. 1, 2008, pp. 2-13. https://doi.org/10.1016/j.cma.2008.05.004.

[17] Sabater, C., and Goertz, S., "An Efficient Bi-Level Surrogate Approach for Optimizing Shock Control Bumps under Uncertainty," AIAA Scitech 2019 Forum, American Institute of Aeronautics and Astronautics, 2019. https://doi.org/10.2514/6.2019-2214

[18] Forrester, A. I., and Keane, A. J., "Recent advances in surrogate-based optimization,” Progress in Aerospace Sciences, Vol. 45, No. 1-3, 2009, pp. 50-79. https://doi.org/10.1016/j.paerosci.2008.11.001

[19] Han, Z.-H., Goertz, S., and Zimmermann, R., "Improving variable-fidelity surrogate modeling via gradient-enhanced kriging and a generalized hybrid bridge function," Aerospace Science and Technology, Vol. 25, No. 1, 2013, pp. 177-189. https://doi.org/10.1016/j.ast.2012.01.006

[20] Sobol, I., "On the distribution of points in a cube and the approximate evaluation of integrals," USSR Computational Mathematics and Mathematical Physics, Vol. 7, No. 4, 1967, pp. 86-112. https://doi.org/10.1016/0041-5553(67)90144-9

[21] Duvigneau, R., "Aerodynamic Shape Optimization with Uncertain Operating Conditions using Metamodels," resreport RR-6143, INRIA, 2007.

[22] Liu, D., and Goertz, S., "Efficient Quantification of Aerodynamic Uncertainty due to Random Geometry Perturbations," Notes on Numerical Fluid Mechanics and Multidisciplinary Design, Springer International Publishing, 2014 , pp. 65-73. https://doi.org/10.1007/978-3-319-03158-3_7

[23] Dwight, R., and Han, Z.-H., "Efficient Uncertainty Quantification Using Gradient-Enhanced Kriging," 50th AIAA/ASME/ASCE/AHS/ASC Structures, Structural Dynamics, and Materials Conference, American Institute of Aeronautics and Astronautics, 2009. https://doi.org/10.2514/6.2009-2276

[24] Echard, B., Gayton, N., and Lemaire, M., "AK-MCS: An active learning reliability method combining Kriging and Monte Carlo Simulation,” Structural Safety, Vol. 33, No. 2, 2011, pp. 145-154. https://doi.org/10.1016/j.strusafe.2011.01.002.

[25] Bect, J., Ginsbourger, D., Li, L., Picheny, V., and Vazquez, E., "Sequential design of computer experiments for the estimation of a probability of failure," Statistics and Computing, Vol. 22, No. 3, 2011, pp. 773-793. https://doi.org/10.1007/s11222-011-9241-4 
[26] Schrauf, G., "Status and perspectives of laminar flow," The Aeronautical Journal, Vol. 109, No. 1102, 2005 , pp. 639-644. https://doi.org/10.1017/s000192400000097x

[27] Schrauf, G., "Large-Scale Laminar-Flow Tests Evaluated with Linear Stability Theory," Journal of Aircraft, Vol. 41, No. 2, 2004, pp. 224-230. https://doi.org/10.2514/1.9280

[28] Kulfan, B. M., "Universal Parametric Geometry Representation Method,” Journal of Aircraft, Vol. 45, No. 1, 2008 , pp. $142-158$. https://doi.org/10.2514/1.29958

[29] Gerhold, T., "Overview of the Hybrid RANS Code TAU," MEGAFLOW - Numerical Flow Simulation for Aircraft Design, Springer Berlin Heidelberg, 2015, pp. 81-92. https://doi.org/10.1007/3-540-32382-1_5

[30] Drofelnik, J., and Ronch, A. D., “2.5D+ TAU User Guide,” Tech. rep., DLR, 2017.

[31] van Ingen, J. L., "A suggested semi-empirical method for the calculation of the boundary layer transition region,” 1956.

[32] Schrauf, G., "LILO 2.1 - User's guide and tutorial,” Tech. rep., Bremen, Germany, GSSC Technical Report 6, originally issued Sep. 2004, modified for Version 2.1, July 2016.

[33] Schrauf, G., "COCO - A Program to Compute Velocity and Temperature Profiles for Local and Nonlocal Stability Analysis of Compressible, Conical Boundary Layers with Suction,” Tech. rep., ZARM Technik Report, November 1998.

[34] Schrauf, G., "Industrial View on Transition Prediction," Recent Results in Laminar-Turbulent Transition, Springer Berlin Heidelberg, 2004, pp. 111-122. https://doi.org/10.1007/978-3-540-45060-3_9

[35] Gerhold, T., and Neumann, J., "The Parallel Mesh Deformation of the DLR TAU-Code," Notes on Numerical Fluid Mechanics and Multidisciplinary Design (NNFM), Springer Berlin Heidelberg, 2006, pp. 162-169. https://doi.org/10.1007/978-3-54074460-3_20

[36] Meinel, M., and Einarsson, G. O., “The FlowSimulator framework for massively parallel CFD applications,” PARA2010, 2010. URL https://elib.dlr.de/67768/ 